\title{
Questioning if Macro-Systemic Top-Down Approaches Should Be Used to Develop Smart Cities of the Future
}

\author{
Marian Constantin VASILE and Marian Liviu MOCAN \\ Faculty of Management in Production and Transportation at Politehnica University of Timisoara, \\ Timisoara, Romania \\ Correspondences should be addressed to: Marian Constantin VASILE; \\ marianconstantin.vasile@zalmo.ro
}

Received date: 15 August 2018; Accepted date: 10 January 2019; published date: 8 April 2019

Academic Editor: Oldřich Horák

Copyright (C) 2019. Marian Constantin VASILE and Marian Liviu MOCAN. Distributed under Creative Commons CC-BY 4.0

\begin{abstract}
Cities differ greatly from one to another, even if they serve the same essential purposes. Moreover, they should collaborate at all levels for producing synergistic effects, therefore, their major smart solutions should be standardized or at least compatible, whilst adapted to the local contexts. Cities are systems of systems and contain a large variety of components, each of them presenting different characteristics. Given the vast range of technological choices and the unforgiving overall pressure, governors struggle to implement best specific smart solutions for their cities, by usually adopting bottom-up methods, thus, endangering future possible compatibilities with other local or international systems. This research stresses the importance of top-down smart city deployments, by acknowledging also the local specificities. It investigates the status of relevant international contributions, questioning what kind of approaches should be applied by public administrations. The results point out to complex challenges that exist by revealing the status of standardization proceedings, the authors' perspective on the enabling technologies and their dimensions, as well as a related case study concerning a local community of Romania so as to bring the beforementioned issues to light. The main objective of this research is to highlight the associated problematics and draw attention to the urgent need of a new macro-systemic top-down attitude for developing smart cities.
\end{abstract}

Keywords: smart city interconnecting, international standard, enabling technology, collaborative governance

Cite this Article as: Marian Constantin VASILE and Marian Liviu MOCAN (2019), "Questioning if MacroSystemic Top-Down Approaches Should Be Used to Develop Smart Cities of the Future", Journal of Administrative Sciences and Technology, Vol. 2019 (2019), Article ID 348811,

DOI: $10.5171 / 2019.348811$ 


\section{Introduction}

There are many complex challenges for developing smart cities of the future and they depend on a substantial variety of factors, e.g. economy, sociology, engineering, management, governance. As reflected in
Fig. 1, smart cities must be built, organized, and coordinated in such a way that the overall status-quo, legislation, technology, solution feasibility, and community's acceptance overlap to the highest extent possible, by finding common denominators for reaching greatest positive effects with smallest efforts.

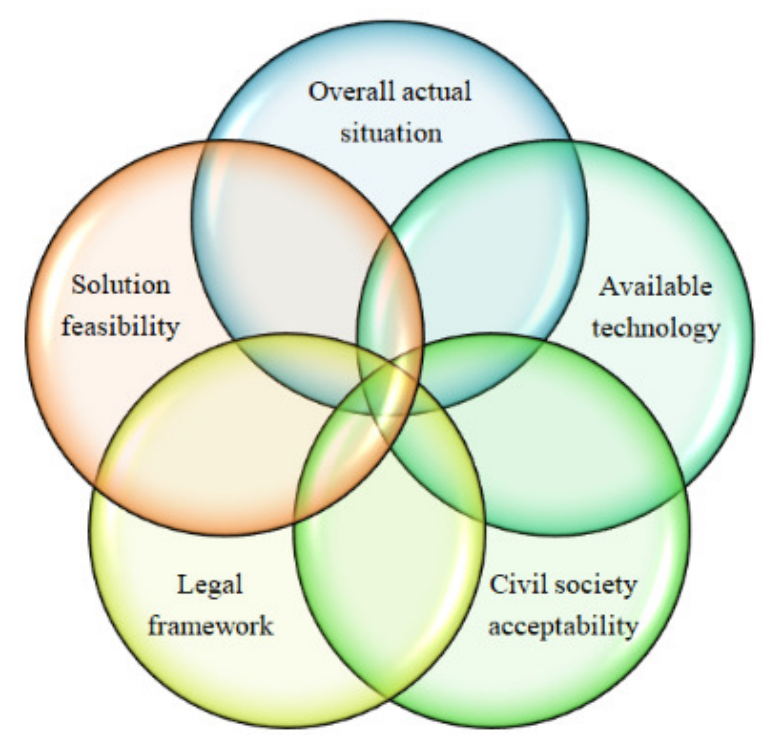

Fig. 1: Common denominators for smart city requirements. Source: the authors

The objective of this paper is to investigate the approach required by cities to successfully implement the best smart solutions in conjunction with their local communities in order to benefit from the right enabling technologies, standardized or compatible systems, and to be able to interconnect with other cities for producing the needed synergistic effects.

\section{Literature Review and Problematics}

This research analyzed the status and evolution trends of smart city systemic elements; mainly digital, to include them in the beforementioned investigation.

\section{Sensors}

Smart city performance and adaptability rely on an interconnected ocean of sensors that are already partially present in the cities of today, which are seen by some authors as "senseable cities" (Ratti and Claudel, 2016, Part III). Accelerometer, temperature, light, micro-electro-mechanical systems, and switch sensors are indispensable in our current applications-e.g. see Fig. 10-, but there are many other types available: barometer, GPS, gyroscope, magnetometer, proximity indicator etc. (Sethi and Sarangi, 2017). Sensors will become more and more versatile, reliable, and accurate, so that 
systems operate at desired performance level including the critical situations, such as emergencies and disasters. These sensors are complemented by humans' direct feedback, which will probably never be completely substituted by any other automatic mechanism in the following decades.

Today's cities are responsible for consuming and manipulating most of world's resources, therefore, they need to implement greener and more efficient day by day procedures, with the help of their sensors specialized in infrastructure, buildings, electricity, water distribution, transportation, healthcare, services, and surveillance etc. (Hancke and Hancke Jr, 2012). As a concrete example, from the public space point of view, Barcelona's Sentilo platform uses more than 1800 sensors to monitor data about energy, noise, urban lab, garbage collection, and parking spots, and this number is expected to increase over time (Sinaeepourfard et al., 2016). Many sensing applications are dedicated to energy efficiency and environmental protection; as these topics are top concerns of the twentyfirst century (see, for instance, Pradeep Kumar, 2016, Merlino et al., 2015, Shamsir et al., 2017).

Sensors have evolved towards a multitude of forms and technologies-including nanotechnology-while adding the new versatile mobility dimension, as in the case of mobile phones, which transform the cities into largely covered, dynamic and interactive entities (Hancke and Hancke Jr, 2012, pp. 395-396). Three sensing platforms are to be highlighted for the ubiquitous coverage: wireless network sensors, IoT systems, and Cloud of Things [ibid, pp. 399-403]. In the case of no existing energy and communications infrastructure, e.g. postdisaster situations, there are already autonomous solutions which acquire and transmit necessary data to the decision makers (Habibzadeh et al., 2017).

Virtual sensors are a distinct important category, which allow simultaneous readings by multiple users in a distributed system. They are simple-to-use programming abstractions of real sensors, which exist locally or distantly-e.g. in the Cloud (Vermesan and Friess, 2014, Chap. 3.7.4)and make the implementation of the Sensingas-a-Service paradigm possible (Vermesan and Friess, 2015, Chap. 8).

\section{Data}

Data collected by sensors are stored, aggregated, processed, distributed, correlated, analyzed, and interpreted in certain schemes, to support human or automatic collaborative decision-making and needed outcomes. These data may be structured-i.e. data is recorded in files with fixed input fields-or unstructured-photos, videos, social media, emails etc. The challenges in managing the data explosion shown in Fig. 2 are multiple, such as ultra-fast acquisition of data, reliable large storage capacity for all circumstances, filtering, prioritization, processing performance, general accuracy, models for interpretation, and information security. Data centers, big data analytics, and cloud computing are few examples of technologies that mostly manage these challenges. 


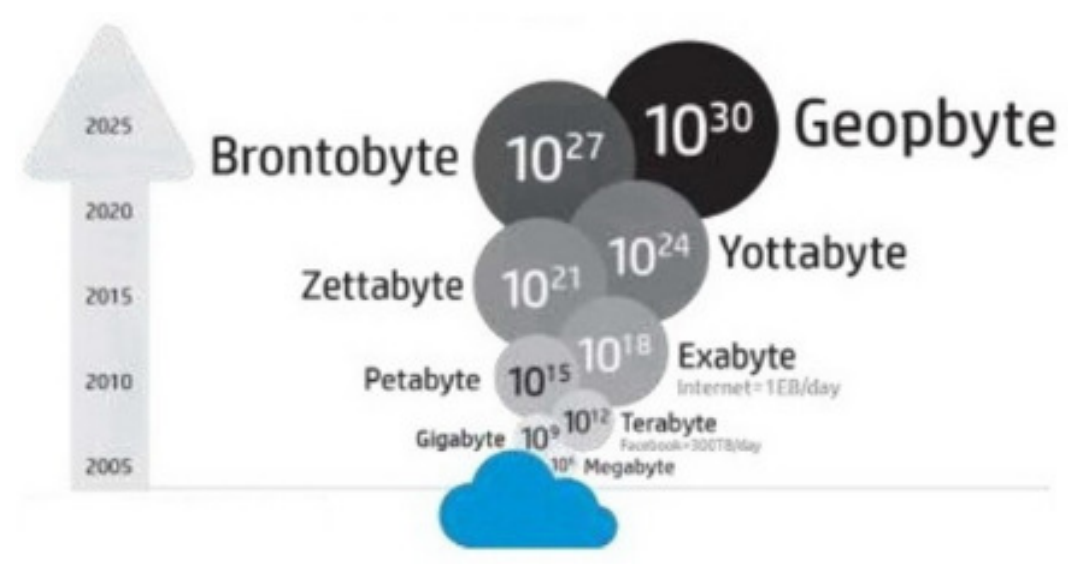

Fig. 2: Estimation of data explosion

Source: Odini (2016)

Data have three main characteristics-volume, velocity, and variety-, they produce value chains within the processes, and trigger human or automatic actions.

Some of main sources for generating data are sensors, smart mobile devices, and web services (Sinaeepourfard et al., 2016). Each of them generates large amounts of data, for example Barcelona's Sentilo platform registers beyond 1.3 million records daily in its data bases [ibid]. These big data are used for predictive analysis, decision automation, data driven businesses, and business performance management (Hashem et al., 2016, Fig. 3), whilst they are systematically processed by smart analyzers, which split, map, shuffle, sort, reduce and deliver all the ingurgitated amounts from the environment [ibid]. To these sub-processes, Khan et al. (2015) add the integration, visualization, as well as supervised and unsupervised learning.

Importantly to underline is that these processing activities happen not only on fixed platforms, but together with mobile platforms which involve smart mobile devices, fogs and clouds (Arfat et al., 2017). To assure interoperability and hide the hardware complexity of many public and private devices operating within a city, more abstraction layers are usually required for the development of applications. For example, iSapiens platform suggests four layers: data, information, knowledge, and wisdom layers (Cicirelli et al., 2017).

Open data, supported by initiatives such as the Open Government Partnership launched in 2011, enable the participation of many actors to faster creation of hard and soft solutions, innovation, synergies, knowledge sharing, and other very positive effects. Last years have imposed new approaches that encourage an active participation of the society, such as crowd sourcing, gamification, co-creation, self-organization, and others (Dijk and Teuben, 2015). For example, Helsinki made available 1030 data bases to the public in 2013, addressing many aspects of the city life, so as to be valorized with these new approaches (Hashem et al., 2016). Moreover, the publishing of these data, facts, and trend lines tempers the population from being suspicious and arguing groundlessly, thus, it promotes a trustful and pragmatic cooperation environment (Goldsmith and Crawford, 2014). Additionally, data democratization and digital access strengthen the social equity (Nguyen and Boundy, 2017), and promote the development of new governance models for smart cities as well (Barns, 2018).

\section{Networks}

Many years ago, scholars have indicated that the evolution of cities is closely linked to telecommunications infrastructure and 
networks in general (see, for instance, Townsend, 2001).

Billions of devices and users are being added each year to the worldwide ecosystem, having more or less predictable communication traffic patterns (Vermesan and Friess, 2015, Chap. 3.6.1). All of them should be digitally interconnected in a ubiquitous, broadband, efficient, scalable, reliable, and self-organizing network at smart city level, which in turn should be connected to the global network. The pressure on the capacity and performance of networks is enormous, regardless whether they are fixed, wireless, mobile, iBeacons, machine-to-machine or IoT (Dijk and Teuben, 2015).

Fig. 3 shows the pressure on the requirements for $5 \mathrm{G}$ communication (Vermesan and Friess, 2014).

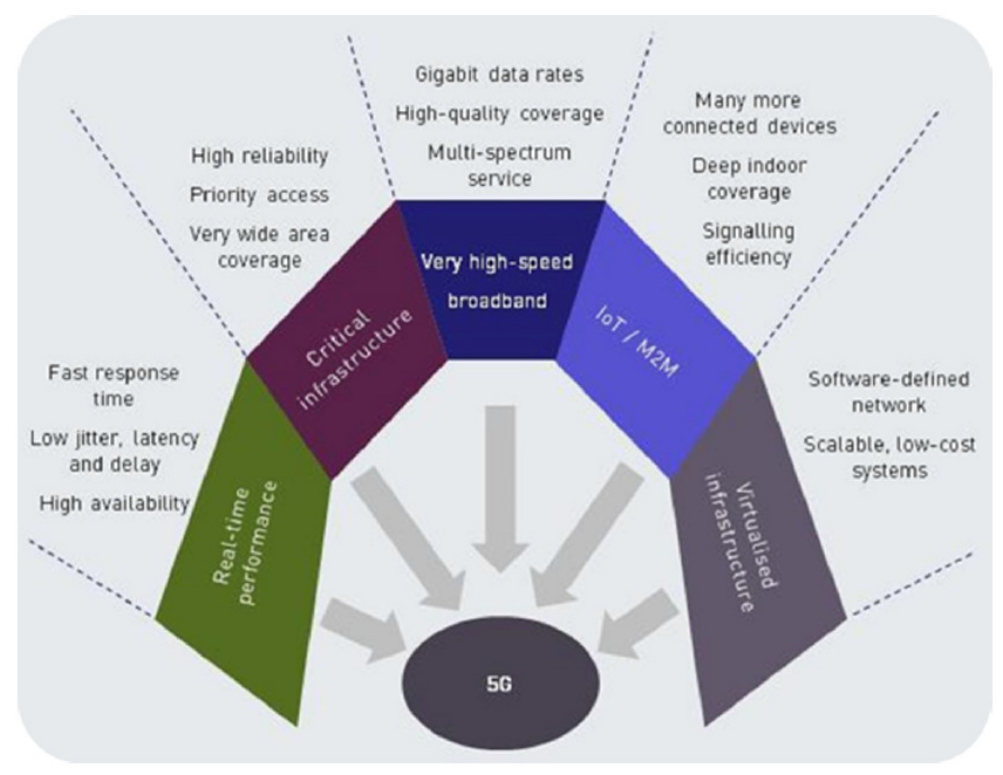

Fig. 3: 5G requirements

Source: Vermesan and Friess (2014)

Networks link people to people, people to machines, and machines to machines in all activity areas: businesses, healthcare, education, governance, public utilities etc. To interconnect this large diversity of entities, standardized platforms must be created and put into the market, by regulating hardware, software, and protocol solutions. Worthy and important to be mentioned is the networking of cities, as well as the networking of services through the Anything-as-a-Service platformsaka. XaaS-, i.e. MaaS, SaaS, PaaS, IaaS, MBaaS etc. (see, for instance, Sneps-Sneppe and
Namiot, 2016). Communication standardswhich are essential-mostly exist (Vermesan and Friess, 2015, Chap. 3.6.2), but there is additionally much to do in the sense of smartly interconnecting everything in this world.

Some institutions make efforts and already propose dedicated platforms, e.g. the Network of Things of the National Institute of Science and Technology-NIST (Strother and Lawrence, 2016). The IoT technologycovered by IoE (Di Martino et al., 2017) and CoT (Sandip and Debabrata, 2017, Mohamed 
et al., 2017)-practically offers limitless possibilities to connect to and interact with any fixed or mobile physical object if it holds a minimal digital compatible interface. Moreover, from another perspective, IoT allows the interconnection of physical, digital and virtual worlds (Vermesan and Friess, 2016, Song et al., 2017). Therefore, most smart cities are based on IoT technology and managed by Cloud, Fog, or other specific platforms, e.g. Sentilo (Sinaeepourfard et al., 2016), iSapiens (Cicirelli et al., 2017), EPIC middleware developed in the context of European Platform for Intelligent Cities (Ballon et al., 2011), and the proposed SmartCityWare (Mohamed et al., 2017).

\section{Processors}

Generally, vendors struggle to produce processors and microcontrollers which are multi-core, fast, versatile, reliable, small, cheap, easy to program, and able to process large quantities of data to incorporate new technologies-e.g. for face recognition in dynamic environments, and to synchronize with other processors and systems.

On the other hand, researches are being undertaken to implement minimum energy and minimum computation devices (Vermesan and Friess, 2014, Chap. 3.5.2.4), which are adapted to the strict specific necessities by considering all usage cases during their entire lifetime.

Depending on usage requirements, today's smart processing is done by combining local IT infrastructures-e.g. PCs-, data centers (Sneps-Sneppe and Namiot, 2016), mobile devices and mobile edge computing (Abbas et al., 2017, Mao et al., 2017), Fogs (Mohamed et al., 2017, Arfat et al., 2017), cloudlets (Pang et al., 2015, Bangui et al., 2017), and the Cloud (Khan et al., 2015, Mohamed et al., 2017, Sneps-Sneppe and Namiot, 2016). The corresponding technologies act locally or distantly, do preprocessing or processing tasks, and are efficient for a limited sum of purposes; therefore, they are usually combined in a tailored architecture to meet the specific set of local requirements. These technologies integrate capabilities that are standalone, part of local area networks or worldwide distributed, performing innetwork(edge) computing and off-network computing (Cicirelli et al., 2017, Fig. 1). According to some scholars, the balance between these two last computing approaches should be thoughtfully addressed and designed (Garcia Lopez et al., 2015).

In certain cases, it is necessary to locally learn from remote devices and users, recognize patterns, and apply inter-device controlling, therefore, the processors must securely communicate, share data and other resources, as well as make use of remote computing power (IEEE Staff, 2016).

\section{Software and Applications}

Software populates and commands almost all intelligent machines on the planet. It may be of various types, structured on different layers, but the main aspect is that it makes the devices "alive", so that they act and react, learn, communicate, and collaborate. Software can be updated; therefore, the devices can be cheaply "reinvented" by keeping the same hardware.

There are different types of software applications, depending on their purpose, capabilities, and localization. For example, some applications are functionally limited, embedded in microcontrollers and perform edge-computing tasks (Garcia Lopez et al., 2015). Some applications are versatile and used locally in larger ICT systems, whilst others "live" in local micro-clouds or the Cloud and are triggered from distance as Software-as-a-Service (Gubbi et al., 2013, Sneps-Sneppe and Namiot, 2016). It is important to do the right job at the right time, in conjunction with the hardware, including the critical environments.

In smart cities, high-level user-oriented applications should cover all activity fields (e.g. transportation, healthcare, energy management), by integrating all necessary enabling technologies, such as Cloud, Fog, IoT (Mohamed et al., 2017, Table 1, Arfat et al., 2017), mobile devices (Sneps-Sneppe and 
Namiot, 2016, Arfat et al., 2017), sensing technology (Shamsir et al., 2017), big data (Khan et al., 2015, Hashem et al., 2016), and cognitive computing (Hurwitz et al., 2015, Vermesan and Bacquet, 2017), etc. For example, a substantial list of such applications in the sustainability area is enumerated by Angelidou et al. (2017): Everimpact, Metropia Synergy, WaterSmart etc.

A special software is the middleware, which is a virtualization bridge between the hardware-specific layer which widely differ from one vendor or technology to another, and the end-purpose applications which are the main target for the developers who want to make the devices intelligent so as to fulfill their tasks. To do that, today's middleware has to offer the developers smart APIs which must be versatile, simple to use, and can enable fast deployments. By using APIs, the integration of devices, networks and applications is facilitated. It is also worth to mention the abstract digital virtualization layers that operate in multiple IoT architectures and platforms-e.g. FIWARE-, as well as the smart cities dedicated operating systems which integrate IoT solutions and various public services (Vermesan and Friess, 2015), but they are far from being mature enough or even not to exist in many cases. A concrete example of an organization that adopted such a vision and solution is the City Protocol Society. Based on its own functional platform and the Open \& Agile Smart Cities initiative (OASC), it develops a network of more than 50 smart cities using FIWARE architectures (Bhatt et al., 2017). Exemplifications of other smart city related middleware in conceptual, implementation, or functional phases are Sentilo (Sinaeepourfard et al., 2016), iSapiens (Cicirelli et al., 2017), GAMBAS (Apolinarski et al., 2014), Arrowhead (Jokinen et al., 2016), EPIC (Ballon et al., 2011), and SmartCityWare (Mohamed et al., 2017). Fig. 4 depicts the principles for organizing SmartCityWare middleware.

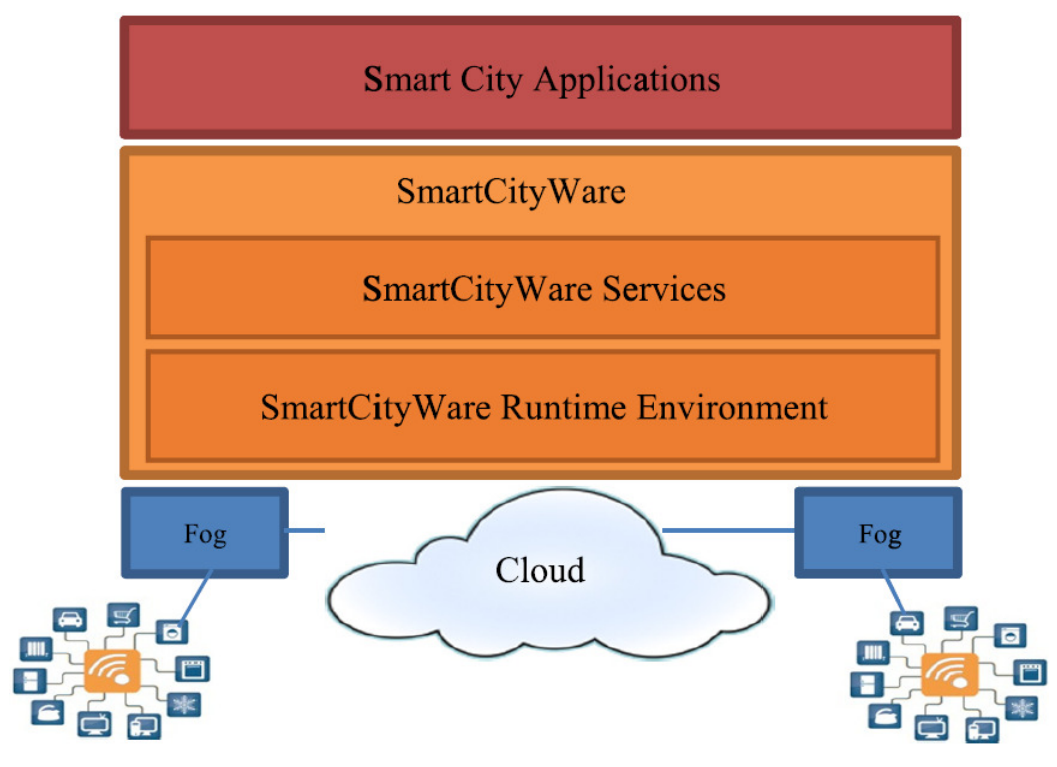

Fig. 4: Middleware of SmartCityWare. Source: Mohamed et al. (2017)

In order to be compatible, all applications middleware of smart cities have to follow imposed guidelines, e.g. to adopt clear roles as 
mirror, boundary, infrastructural, operational, and service agents (Cicirelli et al., 2017).

Finally, a special subject to be highlighted in this subchapter is the Artificial IntelligenceAI-(Nilsson, 2014, Pan, 2016), which evolves based on a special kind of software and does special actions like perceiving, interpreting, communicating, learning, making decisions, and triggering actuations in the real world. AI has already started to be used also in the smart cities (see, for instance, Mathur and Modani, 2016), in various fields, e.g. transportation (Agarwal et al., 2015), public and private buildings (Adio-Moses and Asaolu, 2016, Skouby and Lynggaard, 2014), energy (Mrówczyńska et al., 2014) etc. Great successes are yet to come in this area, although one must say they induce definite risks to humans, therefore, they must be managed carefully.

\section{Actuators}

Actuators change the status of the environment by influencing its components and/or parameters (Sethi and Sarangi, 2017). They are devices powered by any type of energy and vary from very simple ones-e.g. on-off switches (see Fig. 10)- to very complex-e.g. control systems of robots (see, for instance, Vo et al., 2018). One could further extend this standard definition by including AI, machines, and even humans that trigger actions to satisfy requests or necessities, regardless whether they are in the physical or virtual reality.

Like in the case of virtual sensors, virtual actuators control a set of real-life actuators, being triggered by stimuli of the physical or virtual world. This could imply the usage of dedicated middleware to manage and facilitate the interaction between the hardware itself and the higher level services (see, for instance, Merlino et al., 2015).

Today, many actuators are remotely controlled, therefore, they have to be integrated into various platforms of smart cities, e.g. Sentilo (Sinaeepourfard et al.,
2016) and SmartCityWare (Mohamed et al., 2017, Fig. 1).

\section{Electric Energy}

Anything in this world works by using energy. Its production, transmission, distribution, storage, and usage critically influence the entire environment. Humankind has acknowledged this very tight influence, sometimes with negative impact, therefore, all energy subsectors must become smart. "Smart energy aims at greener energy generation, lower energy consumption, an energy consumption pattern with flattened peaks, and a resilient distribution grid" (Dijk and Teuben, 2015).

To implement this vision, following approaches and instruments should be mixed and harmonized to the highest extent possible within the city premises: distributed generation with renewable sources, smart grids, microgrids, smart metering, lower usage through gamification, responsive devices, seasonal thermal energy sources, excess heat use, electric vehicle charging, power of combining, and cooperation in energy markets (Dijk and Teuben, 2015). These aspects apply to all areas of smart cities: buildings, green spaces, mobility, healthcare, public services etc. A vast international literature addresses all these aspects, in conjunction with the smart city topics or not, as smart energy is one of the most pressing themes at worldwide level (see, for instance, Connolly et al., 2016, Helfert et al., 2016, Casini, 2016, Papa and Fistola, 2016).

In the new Energy Cloud concept introduced in 2015 by Navigant Research (see Fig. 5), IoT plays a central role and constitutes a framework for "clean, distributed, and intelligent energy systems. IoT technologies are well-suited for integrating the two-way flow of power, the intermittency of wind, and solar, and behind-the-meter processes such as onsite storage or EV integration" (Strother and Lawrence, 2016). 


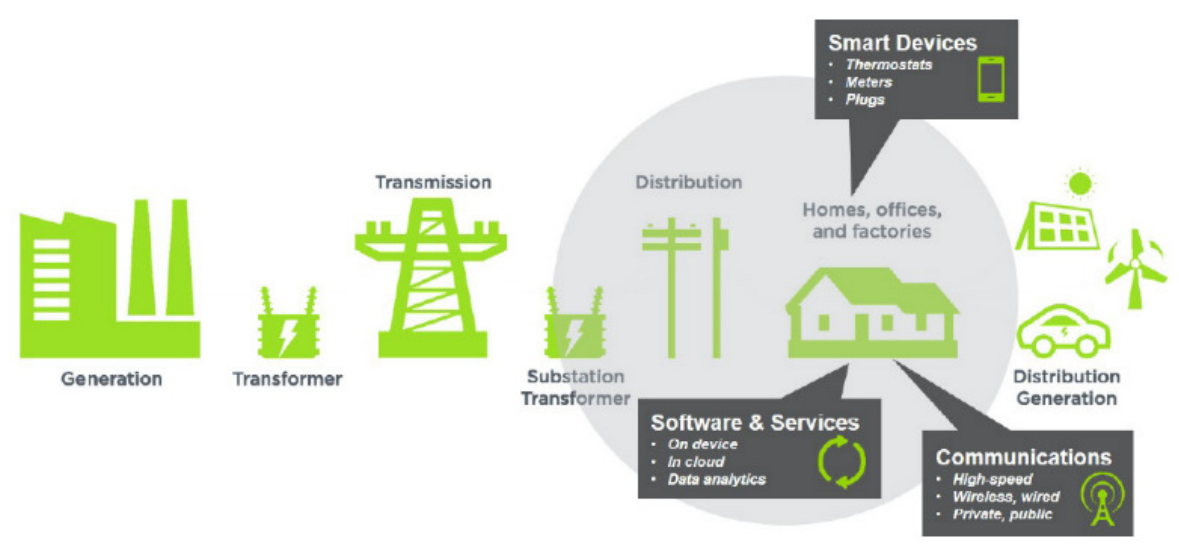

Fig. 5: Elements of the Energy Cloud. Source: Strother and Lawrence (2016)

Another term to describe the energy management system of the future is the "Internet of Energy" (see Fig. 6), which implies a "networked Smart Grid that is largely automated, by applying greater intelligence to operate, enforce policies, monitor, and self-heal when necessary. This requires the integration and interfacing of the power grid to the network of data represented by the Internet, embracing energy generation, transmission, delivery, substations, distribution control, metering and billing, diagnostics, and information systems to work seamlessly and consistently" (Vermesan and Friess, 2014).

Important concerns are related to "energylimited IoT" (Vermesan and Friess, 2014, Chap. 3.5.2.4) and "device level energy issues" (Vermesan and Friess, 2014, Chap. 3.9). 


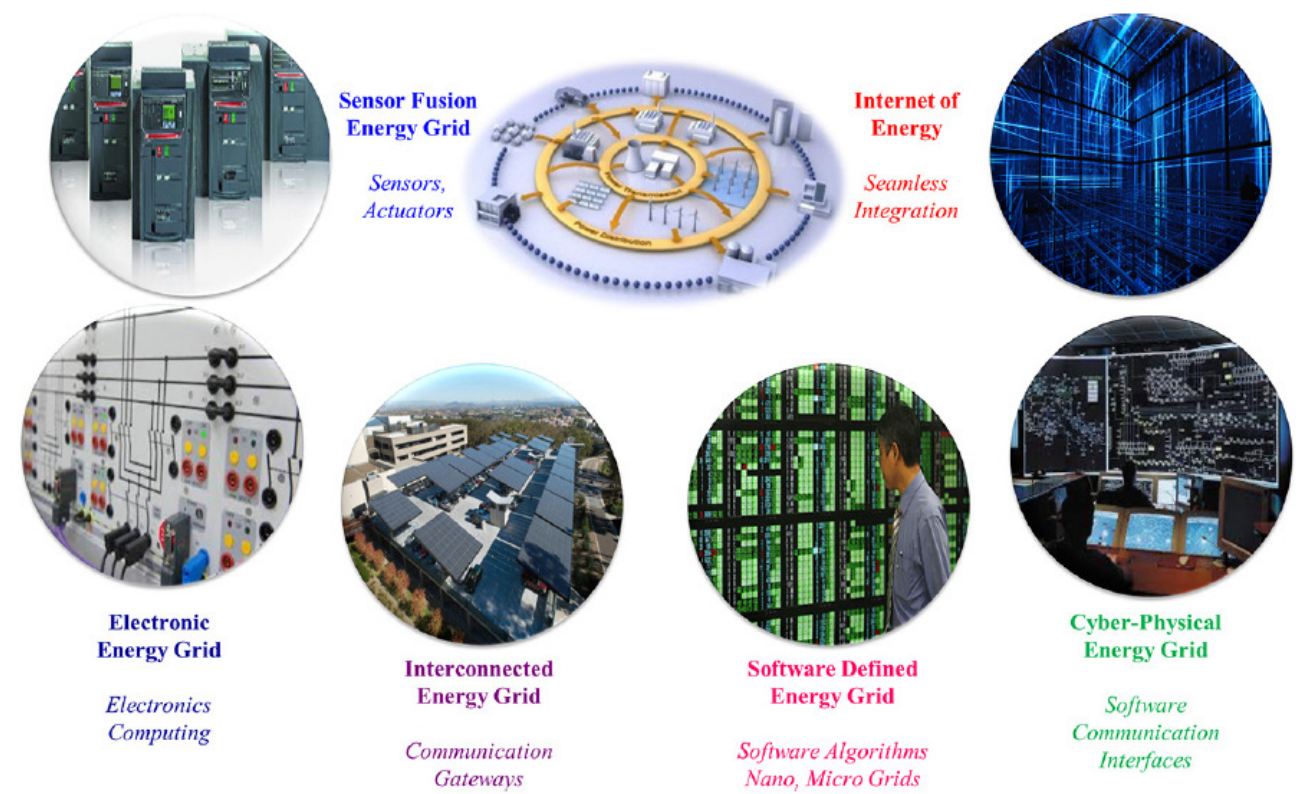

Fig. 6: Internet of Energy concept. Source: Vermesan and Friess (2014)

To be highlighted herein are the energyrelated research directions for autonomy, production self-capacity, conversion, trading platforms, automatic purchase, optimum usage, standardization of new technologies, harvesting of mechanical, thermal, radiant, and chemical energy, along with general power management [ibid, Chap. 3.9], as well as the issues and effects of deploying largescale energy systems managed by ICT infrastructure (Song et al., 2011).

Last aspect to be mentioned is the progressive introduction-with good resultsof AI in the management of all energy subfields (see, for instance, Mrówczyńska et al., 2014).

\section{Human Factor}

Humans are very special actuators of the environment and cities.

A smart society is an inclusive and participative community, which puts everyone's qualities into value. Population of cities should develop towards smart societies, supported in their day by day life by smart solutions and digital public services (Kar et al., 2017, Chap. 5), implying that people's personality and roles are analyzed and valorized accordingly (Kar et al., 2017, Chap. 3 ), along with improving the mindsets of individuals, organizations and communities themselves (Kar et al., 2017, Chap. 13). Smart technical solutions must support today's citizens, but they are unpredictable, reluctant, and not skillful in all domains, as well described by Alexander et al. (2016). Therefore, technical solutions should be error proofed and as user friendly as possible, even if information, options, devices, and systems are more and more abundant and complex.

To increase the prosperity of cities in every sense, all actors should be constructively involved-desirably by digital platforms (Goldsmith and Crawford, 2014)-, including methods like civil hacking (Townsend, 2013). New ways to attract, train, involve, and change behavior of humans are the innovative methods such as gamification, crowd sourcing, co-creation etc., which produce better and better results every year (Dijk and Teuben, 2015). The involvement will evolve over time towards e-democracy, which is based on principles like e-community, egovernance, e-budgeting, e-policy making, e- 
voting etc. (Kumar, 2017), all of them have to be treated cautiously and competently to avoid unpredictable unwanted effects.

People-to-machine interfaces are very important to make life easier and more efficient. They may be of many types, ranging from wearables to stand-alone instruments, and developed by various interaction technologies-voice, video, touch, keyboard etc.

\section{Platforms, Models, Processes, Standards}

In all activity fields, there are numerous and ever-changing visions, policies, strategies, methodologies, use cases, models, platforms, and standards that are available or being imposed at different levels. For example, Fig. 7 indicates today's tendency to transform the ICT network paradigm.

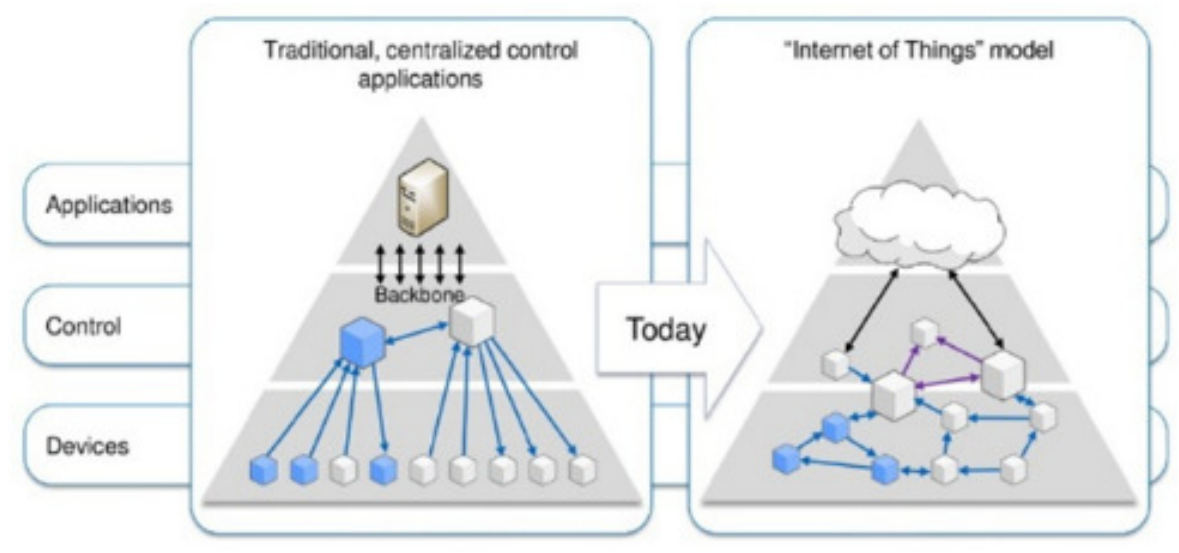

Fig. 7: Rearrangement of ICT network structure. Source: Vermesan and Friess (2014)

It is clear there will always be a diversity of approaches from one community to another, from one city to another, from one state to another, depending on their general status, culture, population, business needs, and governmental maturity. This diversity is produced by multiple top-down and bottomup development approaches, depending on a mix of influencing factors. But there must be both a "common language" and solutions to interconnect all implemented systems, to facilitate collaboration and prosperity. Unfortunately, common solutions could sometimes mean bigger risks, e.g. concurrent hacking threats (see, for instance, Vermesan and Friess, 2015, Chap. 6).

In the meantime, standards of international and national bodies evolve in all domains and cover architectures, data management, activity flows, non-functional aspects etc. Their benefits are obvious, if they are correctly applied (Trivedi, 2016). They reduce risks and invested money, determine faster integration and synergies with similar entities, and allow simpler maintenance etc. It is self-understood that these standards produce diverse results from one case to another in terms of aesthetics, features, and performances etc., but finally the outcomes should be mostly compatible.

At the same time, smart cities need similar models for financing, development, general management, and putting public and private businesses together (Beurden et al., 2017, Ferrer et al., 2013b, Ferrer et al., 2013a, Orłowski et al., 2016).

Development and operation platforms, including those with open access, are necessary for the efficient and effective functioning of communities (Ballon et al., 2011, Sinaeepourfard et al., 2016, Cicirelli et al., 2017, Mohamed et al., 2017, Walravens and Ballon, 2013). They should be easy to use 
and valorize, configurable, autonomic, scalable, upgradeable etc. From processes point of view, these platforms may be much different from one organization to another, as they are defined and controlled by humans, but-in any case-they have to be adapted to the new trends, e.g. to become mobile, datacentric (Dijk and Teuben, 2015), adaptive, event-driven, highly distributed, as well as capable of dealing with unreliable data/resources or working in volatile environments (Vermesan and Friess, 2014, Chap. 3.6). At the same time, these new principles should be standardized and framed, e.g. as Fig. 8 suggests for IoT landscape.

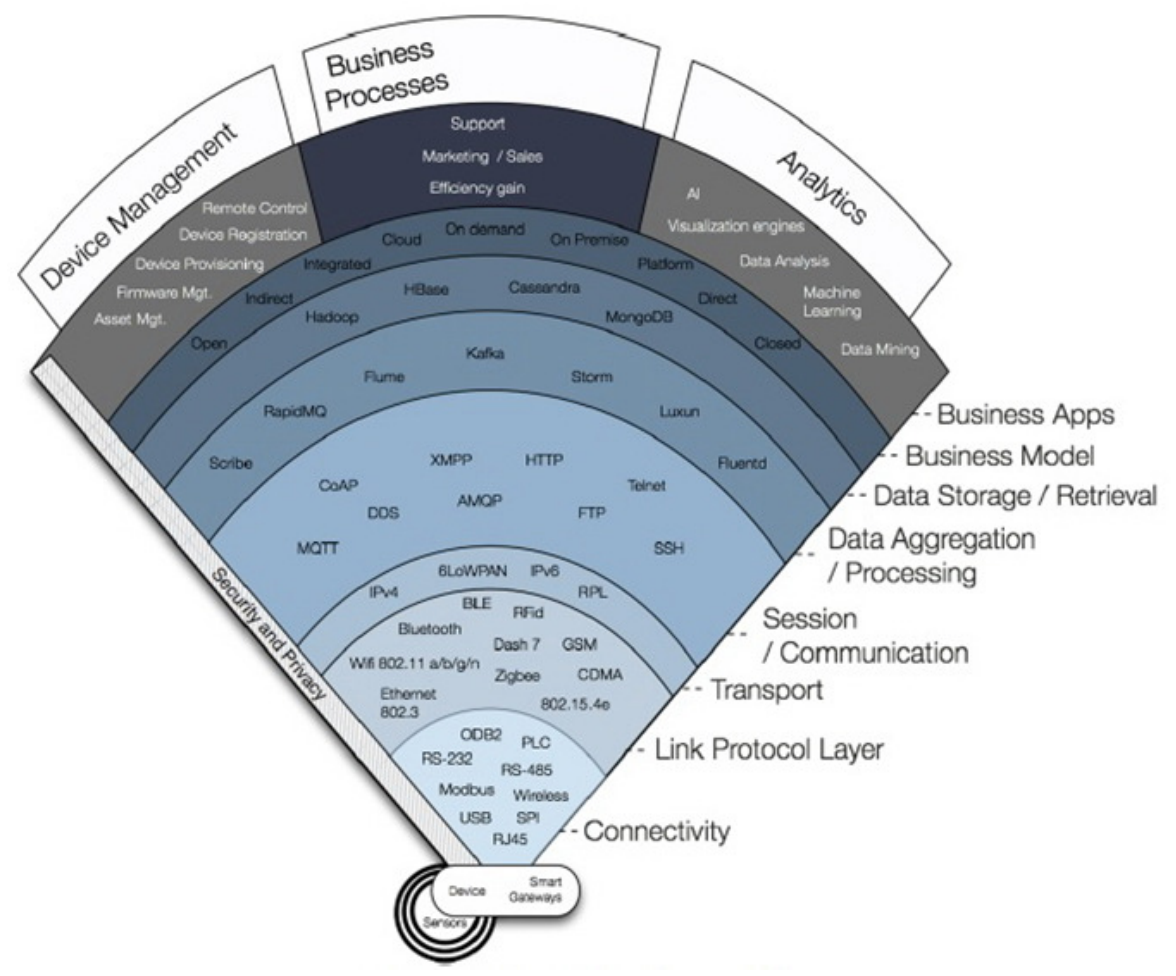

Fig. 8: IoT research and innovation directions. Source: Vermesan and Friess (2014)

Of course, there will always be front runners who will impose new innovative trends at large scale-e.g. digital economy, shift to subscription-based business models, shift from possession to use, shorter product life cycle etc.-and followers who will have to adapt to these new trends (Dijk and Teuben, 2015).

\section{Systems and System Integration}

Cities can be automated and improved almost indefinitely within the limits of available resources and community interests, while maintaining respect for the environment, tradition, and posterity.

Depending on the field of activity and usage, future smart city systems must provide open data and be global and inclusive by connecting people, businesses, machines, knowledge, and other cities as well. One of the Main objectives is to have comprehensive cyber-physical highly-intelligent systems to cover most of the needs and opportunities, by integrating data, sensors, networks, processors, applications, actuators, processes, 
business models, energy sources, and humans. More and more, future systems will also integrate distributed intelligence, software-defined communications, multipurpose networks, cloud/fog/cloudlet computing, data analytics, and batterypowered edge devices. All these require competent planning, synchronization, and management (Beurden et al., 2017, Orłowski et al., 2016).

This philosophy implies many challenges: standardization and certification of products and services, innovative low-cost solutions for handling the fierce global competition and the pressure of current necessities,
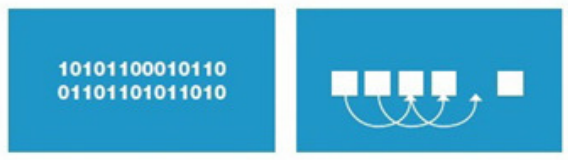

Open Data Infrastructure

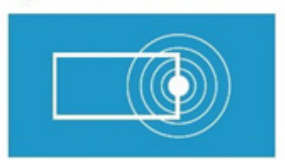

Edge Processing
Modular \& Interoperable

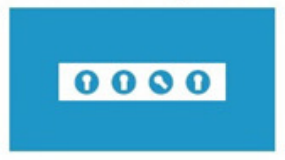

Secure

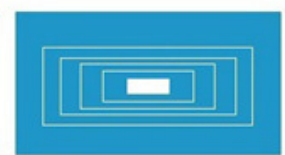

Scalable

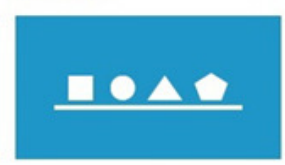

Multi-functional

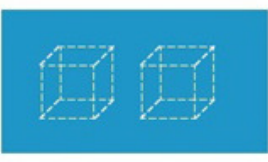

Transparent

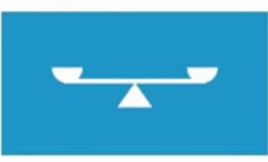

Ethical

Fig. 9: System characteristics. Source: Arup \& Qualcomm (2016)

Efforts are done to frame the technology, e.g. as for the above-mentioned Network of Things model developed by NIST (Strother and Lawrence, 2016), which defines five elements that compose the IoT systems: sensors, communication channels, aggregators, Cloud, decision triggers, and external utilities (see Fig. 10).

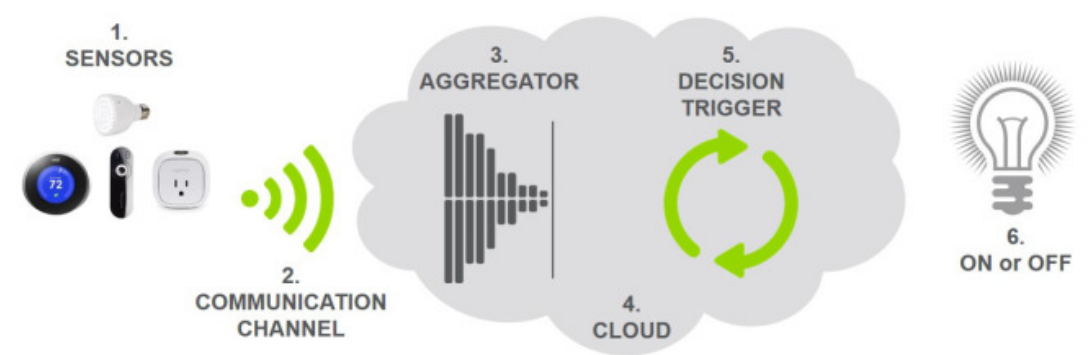

Fig. 10: NIST's Network of Things model. Source: Strother and Lawrence (2016) 
The ability to innovate and bring together individual solutions into aggregates-e.g. public lamp posts with integrated facilities for telecommunications, security, traffic management, and ecologic management-will significantly reduce the cost, space, and speed of implementation (see Fig. 11).

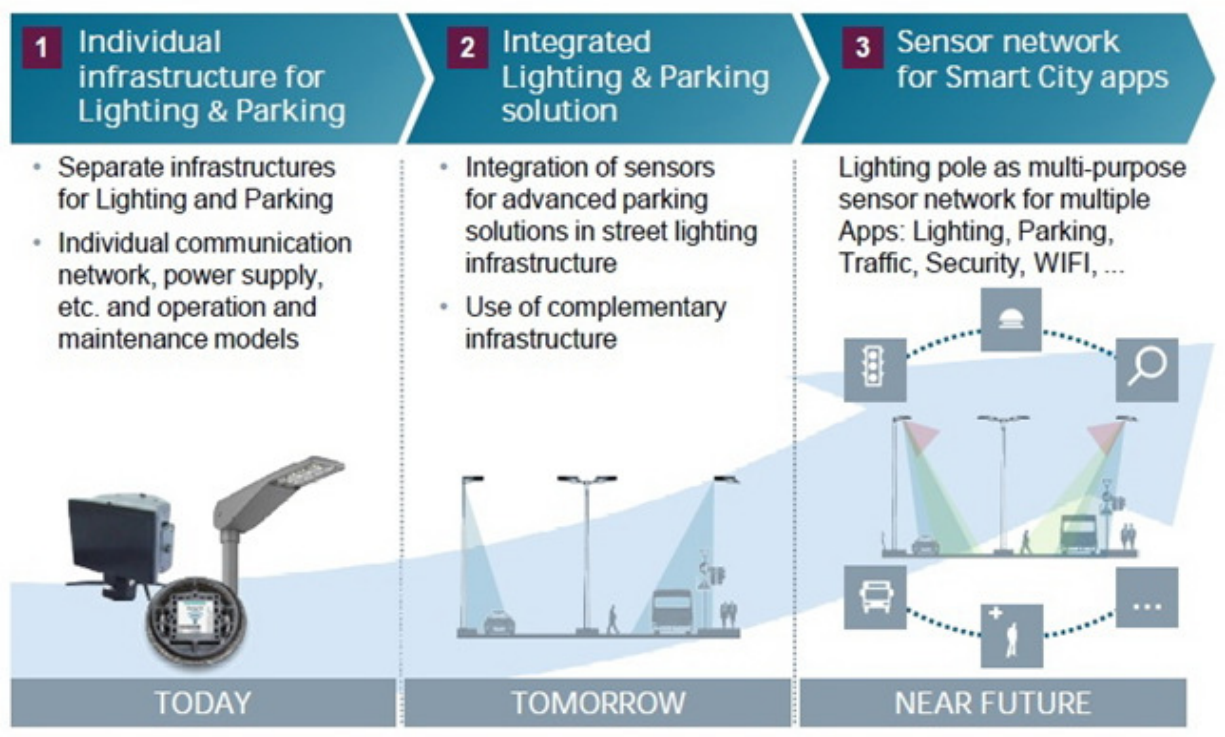

Fig. 11: Multifunctional sensor network. Source: Hessel (2014)

Integrators have an important role in the development of smart cities (AFNOR, 2015, Chap. 7.4.2). The multitude of solutions have to be harmonized with the smart city objectives, services, technologies, management models, and introductory phases (European Telecommunications Standards Institute, 2015, Fig. 2), along with the harmonization of these elements with the nature itself (Ahuja, 2016). All interoperable layers, domains and zones must be made compatible and integrated (see, for instance, European Telecommunications Standards Institute, 2015, Fig. 12). This requires the involvement of all stakeholders and the societal adoption, as well as experimenting pilot projects which are improved in closedfeedback loop (Raaijen and Daneva, 2017, Fig. 2). A suggestive concrete example which covers most of these aspects is the situation of Spanish cities in the pre-crisis period 20002008 and the subsequent development vision for 2030 (Fernández-Güell et al., 2016).

\section{Systems Security, Safety, and Trust}

Today's risks associated with terrorism and cyber-attacks of different natures (see Fig. 12) determine the necessity to better secure the mechanisms that operate in smart cities. Systemic vulnerabilities related to infrastructure-energy, water supply, healthcare, telecommunications, transportation, etc.-, economy and social services are not permitted and can lead to chaos in few moments. This is the perception of city residents too, as highlighted by a significant survey realized by Osborne Clarke (2015). The negative effects could be multiplied, if we suppose that citizens lose a part of their ability to adapt and react rapidly in the future because of the automated systems which they become accustomed to. Therefore, all security related aspects must be treated with extreme caution. 


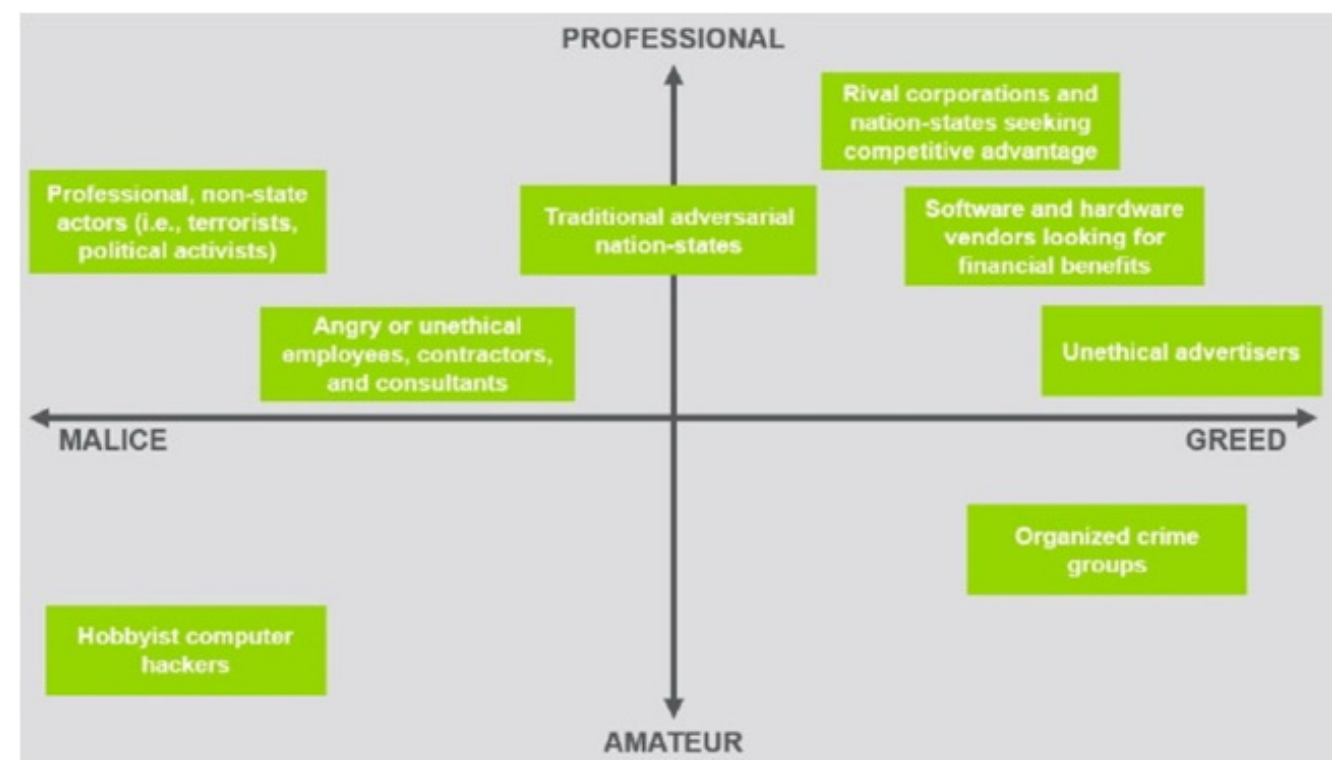

Fig. 12: Cyber-security threat causes. Source: Strother and Lawrence (2016)

Solutions to counteract the cyber-security threats are already in focus since many years, but smart city systems become more and more complex since backdoors and breaching niches conclusively multiply and may be exploited by hackers (see, for instance, Ferraz et al., 2016, International Electrotechnical Commission, 2016, International Telecommunication Union, 2015). All activity fields and areas are targeted and must be safeguarded, e.g. economy (Kumar, 2016), healthcare (Zhang and Shen, 2015), and society categories (Taylor and Rooney, 2016).

From the safety point of view, smart city systems must solve all aspects related to the misuse of public infrastructure and services by humans and machines. At the same time, they have to predict, to be resilient and protective in case of natural/artificial disasters or critical failures (Arafah and Winarso, 2017). These capabilities are not easy to implement, especially that they imply the consideration of many scenarios, as well as additional hardware and/or software.
Concerning privacy, personal and business information must be protected according to user's preferences, e.g. identity, location, and digital operations etc. For this purpose, advances are required with respect to the improvement of cryptographic techniques, privacy-to-design concepts, self-configuring access control, and others (Vermesan and Friess, 2014, Chap. 3.8.3).

All aspects above induce trust or mistrust related to the technologies and solutions that are deployed in smart cities, by guaranteeing their success on the long run or not. Therefore, they must be treated carefully, starting with the requirements engineering phase.

\section{Local Context and Society}

The overall local context-e.g. culture, legislation, resources, political status-is very different from one city of the world to another. Consequently, the success of 
deploying smart city solutions depends on their correct integration into the local setting.

A sensitive related subject is the local society, which should evolve continually into a smarter society, i.e. an inclusive, participative, and collaborative community which puts everyone's qualities into value. The population should be supported in its day by day life by smart public systems and services (Kar et al., 2017, Chap. 5), implying that people's personality and roles are analyzed and valorized accordingly [ibid, Chap. 3], along with improving the mindsets of individuals, organizations and communities themselves [ibid, Chap. 13]. Smart technical solutions must support today's citizens, but they are unpredictable, reluctant, and not skillful in all domains, as described by Alexander et al. (2016). Therefore, technical solutions should be error proofed and as user friendly as possible, even if information, options, devices, and systems are more and more abundant and complex.

To increase the prosperity of cities in every sense, all local actors should be constructively involved in accordance with the principles of collaborative governance (Bolívar and Meijer, 2016, Emerson et al., 2012)-desirably by digital platforms (Goldsmith and Crawford, 2014). Some innovative methods to attract, train, involve, and change behavior of humans are gamification, crowdsourcing, co-creation etc., which produce better and better results every year (Dijk and Teuben, 2015).

\section{Theory and Research Directions}

Given the fast and ever changing social, economic, territorial, political, and natural environments, a mandatory transversal feature of future smart city systems is the capacity to learn, adapt, and respond in real time, irrespective of the implementation solutions. The argument is that, at present, scientists have not yet identified and mastered all algorithms that rule communities and nature. These systems must be capable of permanently monitoring and identifying patterns that exist in the ecosystems and that cannot be framed by simple mathematical models. Over time, they will be able to determine sophisticated algorithms that govern the real world and then they will improve their performance accordingly. Such adaptive systems rely on data accumulation, in absolute and relative terms, targeting various phenomena trends, seemingly chaotic facts-but which could be characterized by fractal patterns for example-, synergetic effects of overlapping causes, human behavior, unstructured feedback of users etc. A consequence of such adapting capabilities is that, depending on the local conditions, an identical smart system could evolve and behave very differently from one world city to another.

To deploy attractive, efficient, effective, and trustful smart city systems, as well as to interconnect these systems to each other and to the systems of other smart cities, compatible solutions must be adopted and implemented. This requires right vision, planning, requirements engineering, implementation, field deployment, operation, maintenance, and upgrades. Therefore, the approach should mostly be top-down by making use of international/national standards and regulations as grounding platform.

In theory, it is possible to standardize the inputs, outputs, interfaces, characteristics, business models, and processes of smart city systems, by covering main aspects concerned: technical, managerial, materials, equipment, and information exchange. It is obvious that all details of smart cities can neither be standardized nor implemented the same way, given the specific local status, resources, existing systems, and politics.

Still, it is essential to urgently create international frameworks for consolidating the future smart cities with as straightforward solutions as possible. Cities are already finding and implementing their own solutions for facing their challenges and therefore, if not providing these frameworks, they will become much different during next decades, consequently, reducing the multiple synergetic effects that could be generated. Therefore, the existent, developing and foreseen smart city standards must be actively acknowledged and promoted (i.e. first research direction of this paper). 
Even if these international frameworks are acknowledged by city administrations, it is furthermore necessary to understand their sense and implications, as well as their related enabling technologies (i.e. second research direction of this paper), which must be synthesized and brought to light by scientific studies.

It is also to be highlighted that regardless of the city business models themselves, smart solutions must be firstly adapted to and validated by the local community and direct stakeholders (i.e. third research direction of this paper), to meet the real expectations and needs, on top of gathering all necessary support for the upcoming efforts. The related results could be surprising, for example some seemingly "low hanging fruits" solutions could encounter multiple blockages, while some tougher-to-implement solutions-e.g. general platforms with middleware and virtualization-could enthusiastically boost the development of the city on the long run. So, future acceptance and implementation feasibility must be-in the first place-very well evaluated, planned, and introduced by pilot experimental projects, in a collaborative approach.

Finally, it is worth suggesting that in our competitive and challenging world, by applying the vision of smartly interconnecting the cities at all levels (i.e. forth research direction of this paper), they would synergistically work together to benefit from opportunities, solve necessities, overcome problems, handle threats, and learn faster from each other. So, this desideratum should be actively supported, researched, and implemented.

\section{Research Methodology}

Fig. 13 points out the inputs and outputs of the methodology used by the authors for this research.

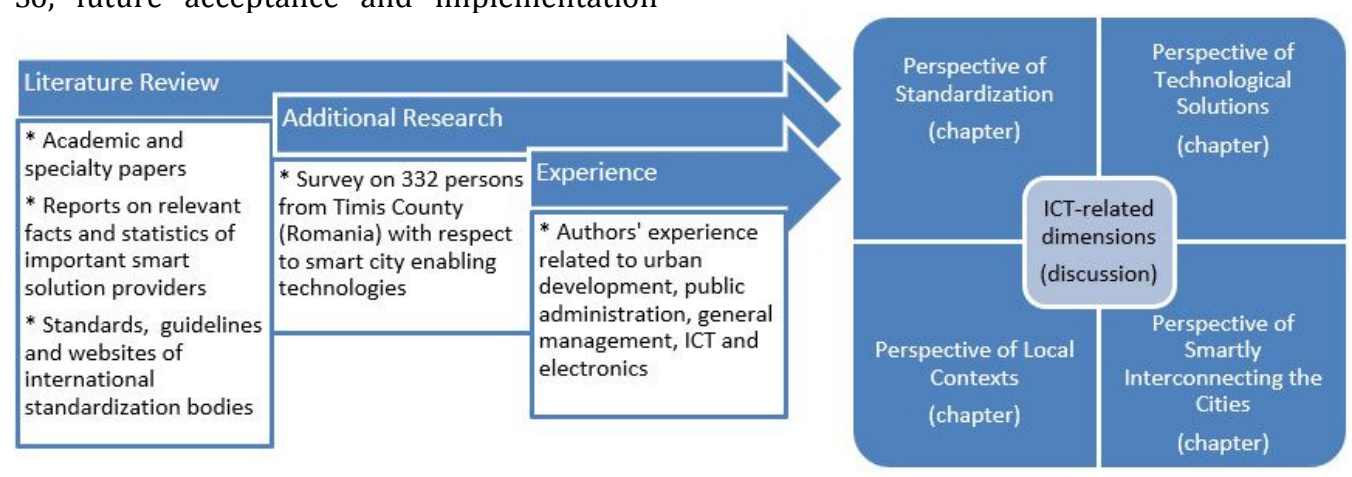

Fig. 13: Methodology inputs and outputs. Source: the authors

Besides their experience, the authors reviewed the international specialty literature and surveyed 332 persons from Timis County (Romania), so as to determine the required outputs of the research, which are highlighted in the following two chapters, together with their subchapters.

\section{Results}

Perspective of Standardization
International standards were published with respect to smart cities and their elements, but not all parts of the puzzle exist, so as to completely shape the future smart cities as systems of systems. Important efforts have been made by international bodies and, in parallel, by the European Commission, CEN, CENELEC, ETSI, and national bodies from United Kingdom, Germany, Spain, China, Korea and so on (ISO, 2015, Chap. 7.2).

Many standards are still under development, some of them being reflected by the International organization for 
Standardization-ISO-in its "Smart cities: Preliminary report 2014" (ISO, 2015). This report underlines the ongoing hard process of synchronizing the international and national bodies which develop concomitantly their

Table 1 indicates up-to-date highlights for the work of ISO, ITU, IEC, and IEEE on subjects that are relevant to the systems and standards of which some are being defined for specialty niches and others are being more generally overlapped by parallel definitions.

activity fields of smart city-e.g. ICT, electronics, and energy.

Table 1: Standardization highlights related to smart city systems and domains

\begin{tabular}{|c|c|}
\hline Org & I stan \\
\hline $\begin{array}{l}\text { Iding } \\
\text { IEC }\end{array}$ & $\begin{array}{l}\text { ISO (2019b) - Sections: } \\
\text { - Sustainable cities and communities: vocabulary and guide to establishing } \\
\text { strategies; } \\
\text { - Sustainable development in communities: descriptive framework; management } \\
\text { system; practical implementation; indicators for smart and resilient cities; } \\
\text { indicators for city services and quality of life. } \\
\text { ISO (2019c) - Standards related to smart community infrastructures: common framework } \\
\text { for development and operation; maturity model; smart transportation; electric power } \\
\text { infrastructure; guidelines on data exchange and sharing; metrics. } \\
\text { ISO (2019d) - Standards related to energy management: energy efficiency and savings } \\
\text { calculation for cities; building energy data management etc. } \\
\text { ISO (2019a) - Standards related to IoT and related technologies: } \\
\text { - IoT: reference architecture; use cases; interoperability for IoT systems; } \\
\text { - Sensor networks: services and interfaces, reference architecture; sensor network } \\
\text { and its interface to smart grid system, generic sensor network application } \\
\text { interface; } \\
\text { - Underwater acoustic sensor network. }\end{array}$ \\
\hline ITU & $\begin{array}{l}\text { ITU (2019a) - L series: four sections related to key performance indicators for smart } \\
\text { sustainable cities. } \\
\text { ITU (2019a) - Y series, sections: } \\
\text { - Next generation networks: frameworks and functional architecture models; } \\
\text { service capabilities and service architecture; interoperability of services and } \\
\text { networks in NGN; } \\
\text { - IoT and smart cities \& communities: infrastructure, connectivity, and networks; } \\
\text { frameworks, architectures, and protocols; services, applications, computation, } \\
\text { and data processing; management, control, and performance; identification and } \\
\text { security etc.; } \\
\text { All supplements: guide for city leaders, master-plan, integrated management, } \\
\text { multi-service infrastructure, ICT architecture, stakeholders' engagement etc. }\end{array}$ \\
\hline
\end{tabular}




\begin{tabular}{|c|c|}
\hline Organization & International standards \\
\hline & $\begin{array}{l}\text { ITU (2019b) - Sections: } \\
\text { - Applications of wireless sensor networks in next generation networks; } \\
\text { - } \quad \text { ICT, environment, and climate change; } \\
\text { - Internet of things (IoT) and machine-to-machine communication (M2M); } \\
\text { - United } 4 \text { Smart Sustainable Cities; } \\
\text { - } \quad \text { Trust in ICT. }\end{array}$ \\
\hline IEC & $\begin{array}{l}\text { IEC (2019a) - Sections related to IEC's specific work on: connecting things, smart energy, } \\
\text { mobility, water, smart buildings, and city services. } \\
\text { IEC }(2019 b) \text { - Section related to projects and publications: city service continuity against } \\
\text { disasters - the role of the electrical supply; smart cities reference architecture } \\
\text { methodology. }\end{array}$ \\
\hline IEEE & $\begin{array}{l}\text { IEEE Standards Association (2017) - Synthesis indicating IEEE's specific standards on: } \\
\text { smart grid, intelligent transportation, eHealth, energy efficiency, IoT, 5G, cyber security, e- } \\
\text { Governance, smart home, and learning technologies. } \\
\text { IEEE Standards Association (2016) - Same as above, from another perspective and } \\
\text { including: smart networking and connectivity, smart buildings, smart technologies-smart } \\
\text { sensors, distributed interactive simulation, modelling and simulation standards, systems } \\
\text { with virtual components, and smart city compliance indicators etc. }\end{array}$ \\
\hline
\end{tabular}

A substantial number of standards are being developed, e.g. those targeted by ISO towards smart community infrastructures, ICT, IoT, performance indicators, strategies etc. or by

Table 1. Additional to the underlined is that some relevant standards aim at component or subsector level, e.g. sensors, processors, software, communication protocols, cybersecurity etc.

There are smart city standardization efforts at national levels too, which complicate the worldwide guidelines harmonization on one hand, but accelerate it on the other. In Europe, for example, most advanced institutions, from this point of view, seem to be British Standards Institution (Joss et al., 2017)-which has begun its efforts early and created the Smart City Institute-, Asociación Española de Normalización y Certificación (Marcos Paramio, 2017)-which collaborates closely with RECI network of Spanish cities-, and Deutsches Institut für Normung. An
IEC for electrical supply continuity against disasters. They can be easily identified by investigating the content behind the references from

important related initiative of the European Commission, launched in 2011, is the European Innovation Partnership on Smart Cities and Communities, which cooperates with CEN-CENELEC-ETSI on the standardization matters (AFNOR, 2015).

Some people acknowledge and underline that the standardization for different parts of smart city systems is missing and have made related gap analyses, e.g. ISO (ISO, 2015, Chap. 8), and ITU (ITU, 2015, Chap. 7), whicheven if published some years ago-are mostly valid, as research results and global consensus are slowly reached by international standardization activities. Same scholars confirm also these existing gaps (see, for instance, Bibri and Krogstie, 2017, Table 2 ). The conclusion is that there are still many aspects to cover and gaps to fill-out for completing the overall landscape of smart city standards. 
At present, one of the most important aspect is probably acknowledging and highlighting the smart city as a system of systems and creating the related regulations. In this sense, Fig. 14 shows the abstraction level for the work of some standardization bodies.

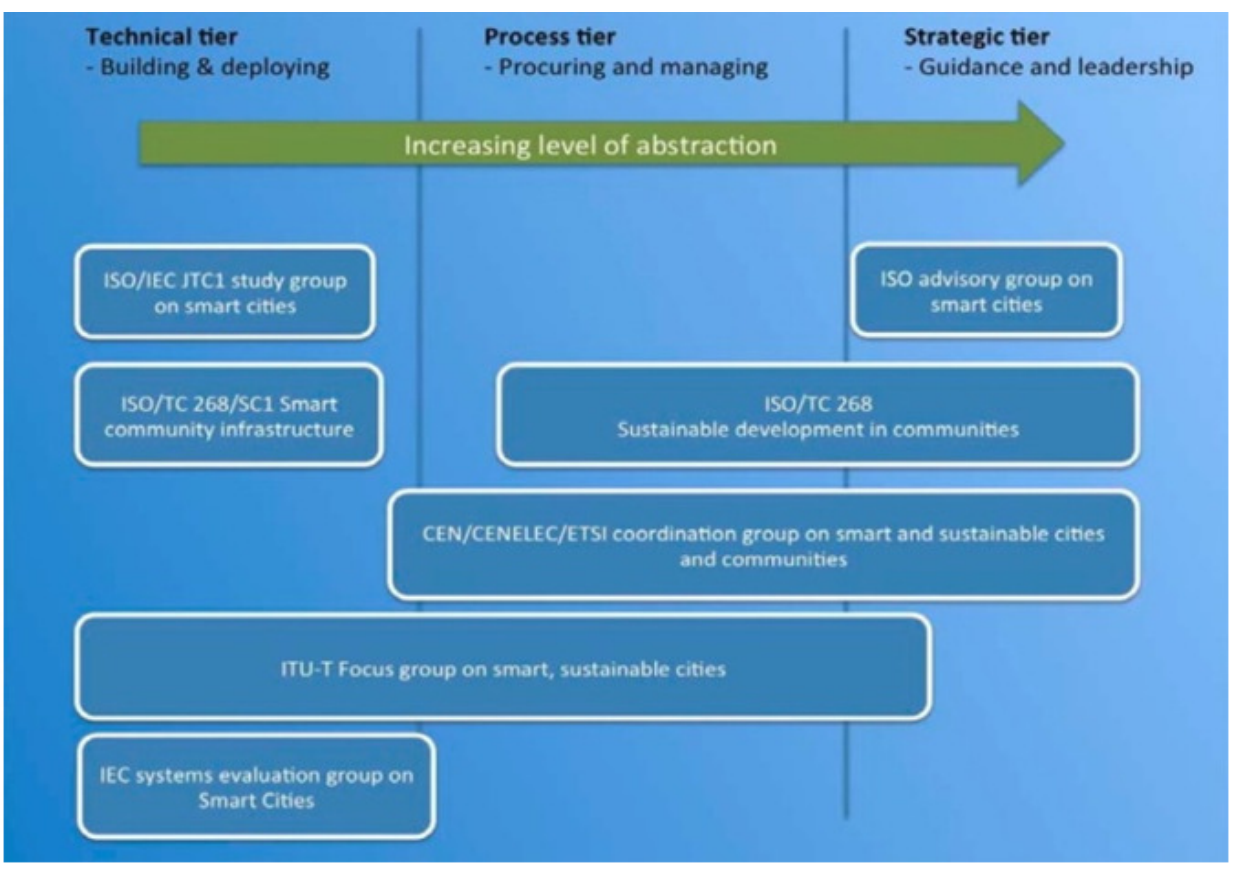

Fig. 14: Abstraction level of standards related to smart cities. Source: Lea (2017)

\section{Perspective of Technological Solutions}

There are many solutions proposed by experts of various fields, communities, public institutions, and private companies, as there are very different realities, needs, and opportunities for cities which decided to become smarter. Under the reality pressure, many of the cities have already adopted and implemented different solutions. Today's main approach is bottom-up, in the sense that each city finds its own way to upgrade itself by sometimes inspiring themselves from other cities and/or by adopting at-hand solutions proposed by private companies which mostly impose-as large stakeholdersthe technological trends and solutions in a top-down manner. The worldwide leading companies offering smart city solutions are benchmarked in Fig. 15, by their strategy-i.e. vision, go-to-market strategy, partners, product strategy, and geographic reach-and

by their execution capacity-market presence, sales \& marketing, product performances \& features, product integration, and staying power (Woods et al., 2017). 


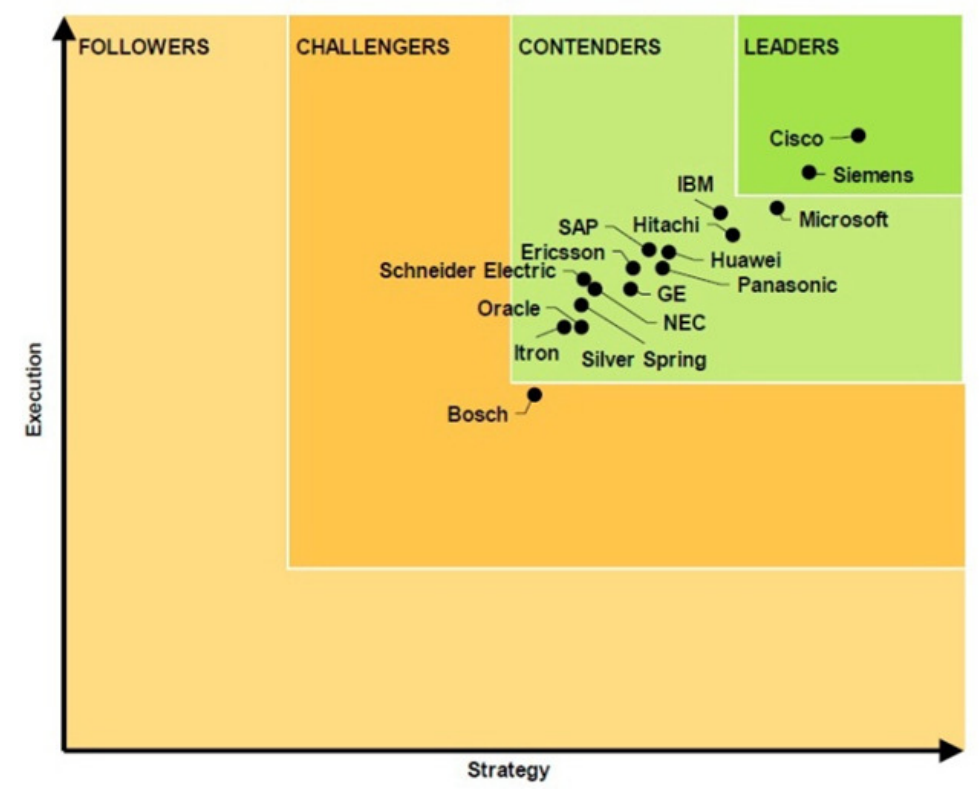

Fig. 15: Leader-board grid of smart cities suppliers. Source: Woods et al. (2017)

As previously indicated, unfortunately some smart city elements and functions are limitedly addressed by current standards, scientific literature, and solution suppliers. Significant examples are those related to the generic middleware, smart adaptive capabilities for all activity fields, and the instruments to smartly interconnect the cities by physical, virtual, and human platforms. This last aspect will probably be the next step to cover, after standardizing, the general concepts, systems, and implementation processes for stand-alone smart cities. Nonetheless, better results could be produced by considering the topic of smartly interconnecting the cities from the very first beginning of the standardization activities and, in parallel and in strong correlation, by the solutions suppliers.
Regarding the enabling technologies for smart cities and as already partly reflected by the previous chapters, the authors determined the following main ones, by analyzing and synthesizing a whole set of specialty literature:

1. Cloud;

2. Internet of Things;

3. Big Data;

4. Broadband Connectivity;

5. Data Centers;

6. Mobile Phones/Devices;

7. Artificial Intelligence, including Machine Learning;

8. Social Media;

9. Online/Web Platforms;

10. Nanoelectronics;

11. (Last generation) Sensors;

12. (Last generation) Actuators; 
13. Renewable Energy Technologies;

14. Smart (environmental-friendly and performant) materials, including the conjunction with cryogenics.

These technologies are most important for developing the smart cities of the future. Therefore, their role and valences must be acknowledged and used as such.

\section{Perspective of Local Contexts}

In accordance with the collaborative governance principles, 332 persons from
Timis County of Romania were surveyed by the authors in July-August 2018 with respect to the 14 enabling technologies mentioned before. This process was applied for identifying local community's perception on future possible smart city solutions and, therefore, for their pre-validation.

The responders were categorized as below (see Fig. 16). Concerning their residency, 21\% did not specify their exact location, while "rural" and "urban" mean localities outside Timisoara's metropolitan area.
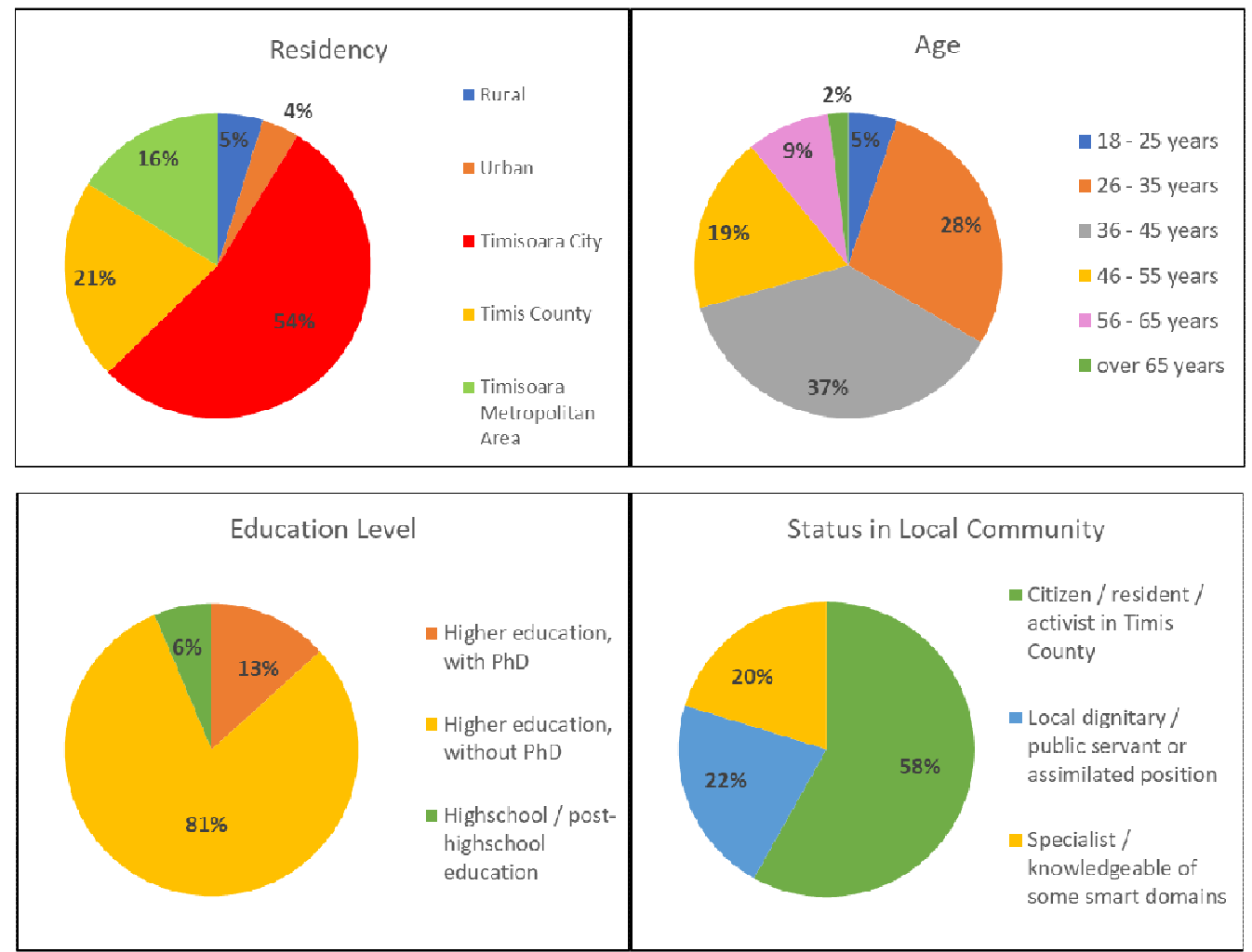

Fig. 16: Responder categories for Timis County's survey related to the 14 enabling technologies. Source: the authors 
The survey results indicate that the responders' majority recommend all enabling technologies as being important for the smart development of Timis County localities (see Fig. 17):

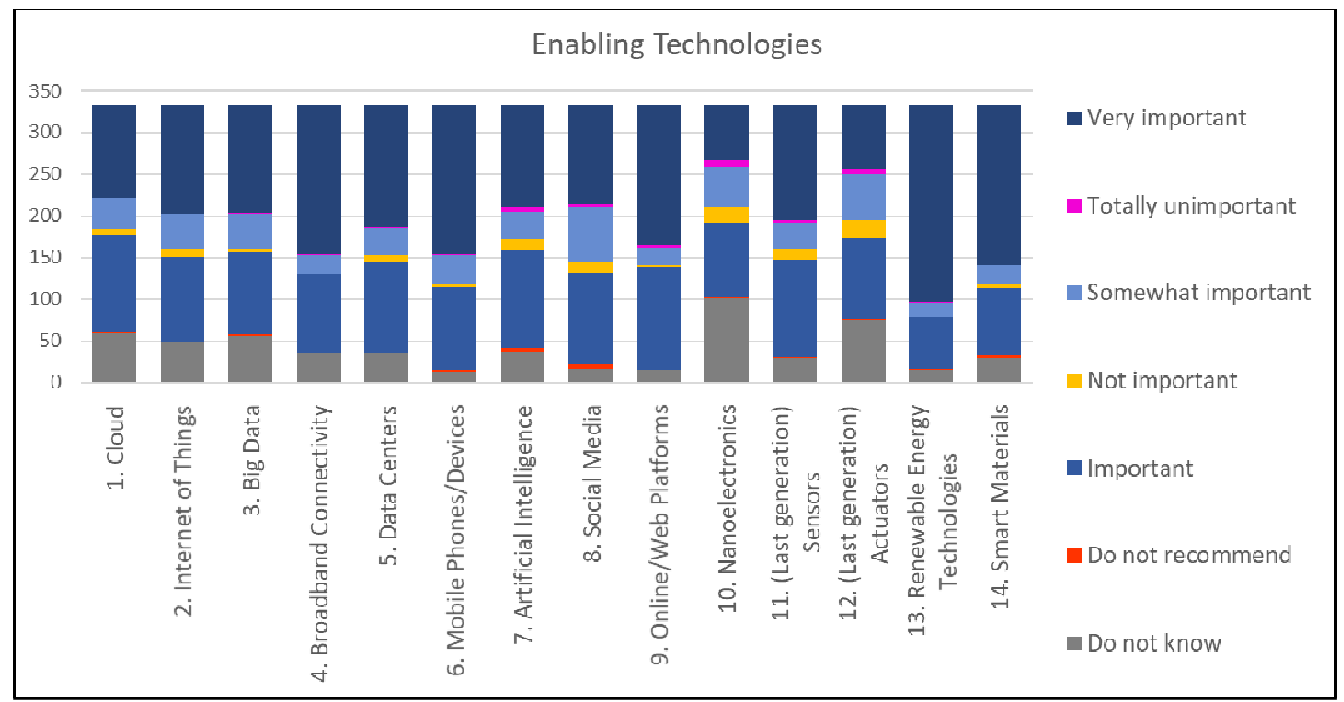

Fig. 17: Survey responders' statement with respect to the 14 enabling technologies.

Source: the authors

To be underlined in the survey is the response of public servants and dignitaries (see Fig. 18), especially that a significant percentage did not know about the five most important smart city enabling technologies, i.e. Cloud, IoT, Big Data, Broadband Connectivity and Data Centers (Huawei, 2017, p. 23).

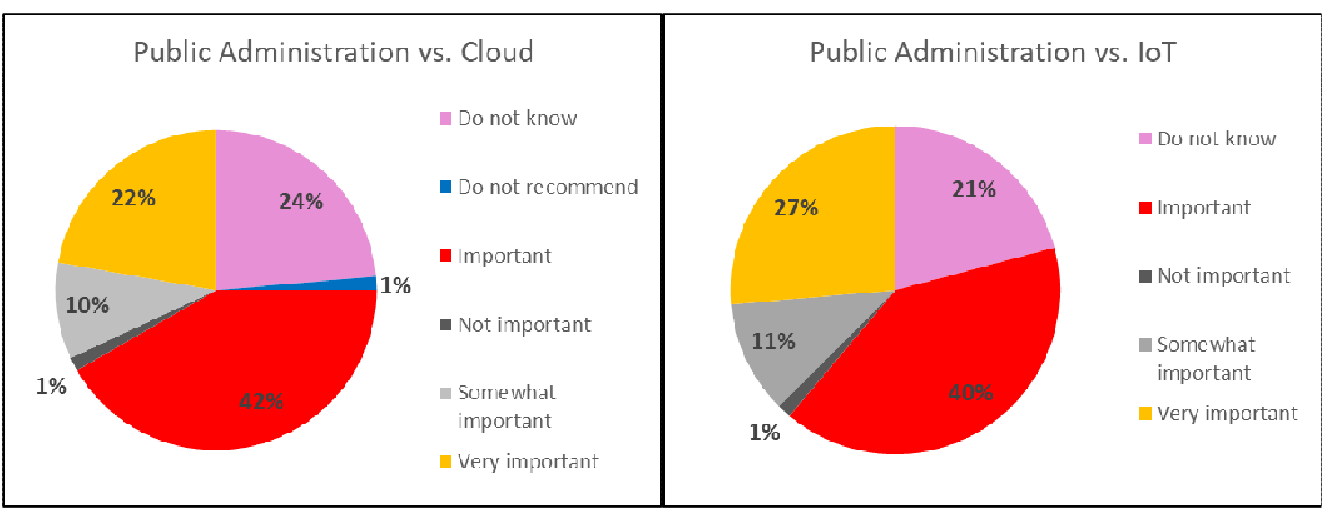

Marian Constantin VASILE and Marian Liviu MOCAN (2019), Journal of Administrative Sciences and Technology, DOI: 10.5171/2019.348811 

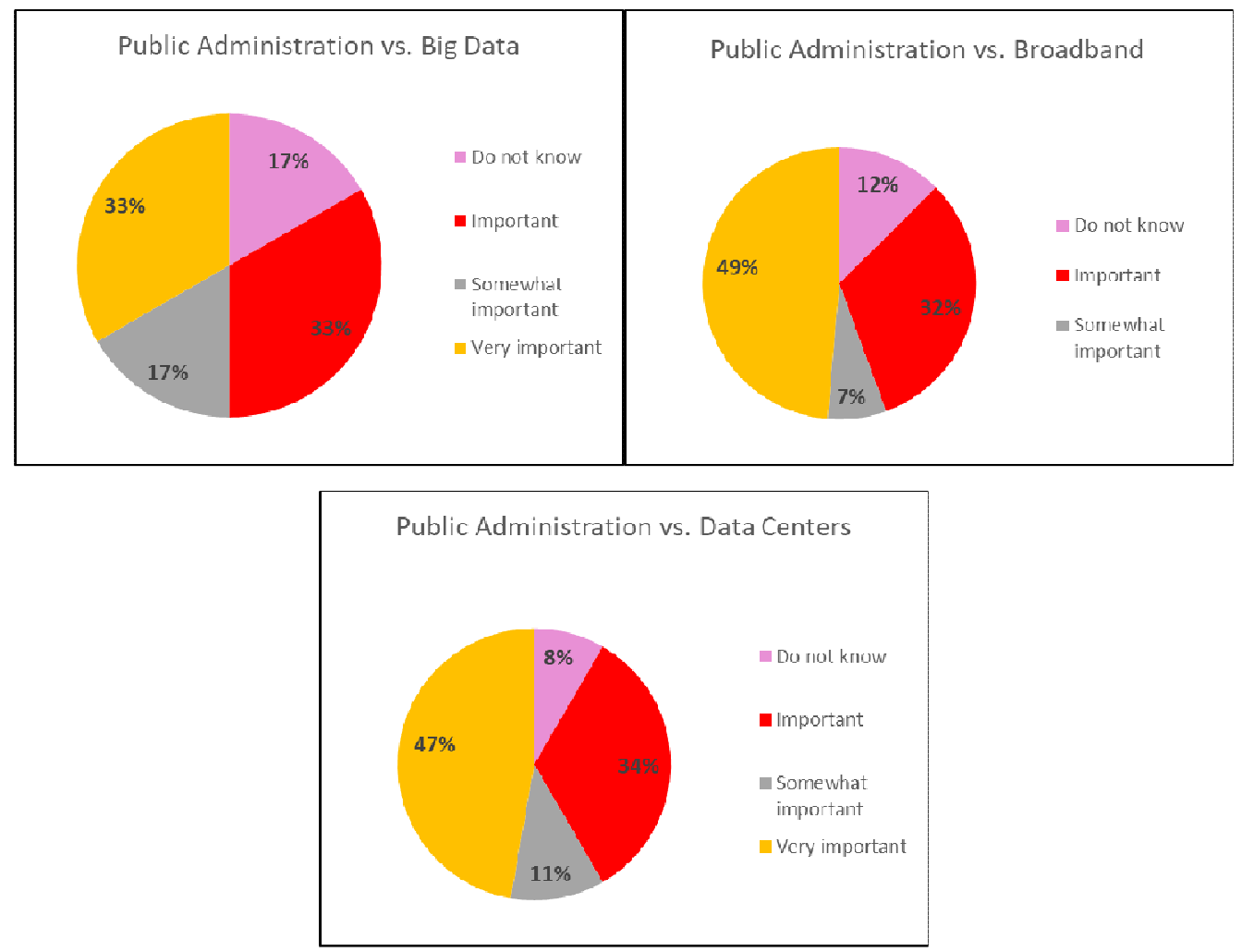

Fig. 18: Statements of survey responders working in public administration. Source: the authors

Of course, all figures in the graphics from above are different from one local community to another and governors must take them carefully into consideration, if they want to be successful with the smart solutions which they are envisioning by themselves, but which could be greatly unsynchronized with the realities from the field. For example, in Timis County, strategies to introduce the enabling technologies in the public administration sector should consider a step by step approach, in order not to disturb the continuity of public activities and create unnecessary problems.

As a general conclusion for this subchapter, this should be further researched, local contexts could be very different from one world city to another, depending on a large variety of factors. This subchapter is only an exemplification. Therefore, to build cities which are smart in the real sense, public planners should firstly investigate the preparedness, perceptions, and positions of their local communities with respect to the enabling smart principles and technologies they want to apply.

\section{Perspective of Smartly Interconnecting the Cities}

With respect to the desideratum of smartly interconnecting the cities, one must say that a rich multidisciplinary literature validates the interdependencies and the need for partnerships between the cities of the world (Sigler and Martinus, 2017, Soler, 2015, Smith and Timberlake, 1995, Palomo-Navarro and Navío-Marco, 2017), but there is much to do in this direction as firmly highlighted by Tranos and Gertner (2012). These two authors drew attention to a poor and limited literature on transnational urban networks and to the necessity to stipulate interurban strategies within smart city policies by highlighting six interdependency categories that occur between the world cities, with respect to ICT technologies, businesses and 
international economy, inclusive society, creative industries, social and intellectual capital, as well as urban sustainability.

It is well known that isolated cities may fall rapidly on a descendant slope, unless they undertake innovative measures to reconnect to the external realities (Tranos and Gertner, 2012, Chap. 4). Therefore, besides the upcoming inherent opportunities, interconnecting to other vibrant cities is a critical necessity for all cities, by paying special attention to the fact that the geography and typology are very different from one functional network of cities to another (see, for instance, Sigler and Martinus, 2017).

At the moment, the subject which is mostly treated with respect to the interconnecting of cities is the network governance (see, for instance, Martínez et al., 2016, Provan and Kenis, 2008), so - at the moment - one cannot talk about "smart networking" (Vasile, 2018).

\section{Discussion and Post-Research Questions}

All fore-mentioned aspects prove that smart cities are very complex entities, composed of many systems that must operate in a smart and sustainable manner with care for humans and moral principles by using and enabling edge technologies.

A Question is which mandatory minimal smart components are to be implemented, integrated, and rolled out by using well streamlined phases, in order for the cities to be able to intelligently face their challenges, so that added value, services, quality of life, security, safety, cost reduction, and sustainability are at desired performance levels? (Q1).

Another question is to which extent smart city systems should be standardized (Q2), given that some international/national standards are already published or under rushed development?

An essential aspect is also that, until all needed backbone standards and regulations are ready, cities cannot wait. Cities are highly pressed by the increasing crowd of people and machines, political changes, globalization issues etc., not to mention the disruptive technologies-e.g. sharing economy, crypto- currency, AI, crowd sourcing, co-creation, XaaS-which emerge and create deep imbalances. Therefore, cities must invest money in solutions that are aligned to future trends and contextual developments. Hence, $a$ natural question emerges, that is how much cities should/could wait for the appearance of standardized smart concepts, related legal decisions, and the concurrent technologies, including the succeeding implementation, field deployment, operational, managerial actions? (Q3)

One conclusion of this research with respect to the international literature and standards indicates a complex multidimensional perspective on the development of smart cities. At this point, in the absence of fullyestablished standardized frameworks and guidelines, it is no wonder that governors are striving to break through the vastness of implementation alternatives-sometimes without understanding all related details and implications-and they are in difficulty to take right decisions. This is one of the reasons why the actual development of cities is bottom-up.

Nine major ICT-related dimensions come to light with respect to deploying smart city systems, which represent the synthetized standpoint of the authors. They are the following:

First dimension (system type):

- Application;

- Integrated multi-application platform;

- Xaas platform for public useIaaS, PaaS, SaaS, DBaaS, MBaaS, MaaS, QaaS etc.;

- System completely managing a specific activity field, with traditional monitoring, controlling, and improving;

- Self-learning self-improving system, containing AI-incl. cognitive computing;

- Complete ecosystem, i.e. harmonized humantechnological-processualenvironmental system. 
Note: all systems from above are defined and characterized by a mixture of following specified dimensions.

Second dimension (reality type): physical, digital, virtual-incl. virtualized objects and augmented reality;

Third dimension (resource type): owned vs. hired, local vs. distant, concentrated vs. distributed, mobile vs. fixed, reliable vs. unreliable etc.;

Fourth dimension (enabling-technology type): Web-incl. next generation Web 3.0-, IoT/IoE/CoT, Cloud/Fog/Cloudlet/mobile edge computing, big data, data center, broadband, AI, nanoelectronics, etc.;

Fifth dimension (data type): open vs. closed, big vs. normal, structured vs. unstructured, reliable vs. unreliable, real-time vs. delayed etc.;

Sixth dimension (process type): user-centric, data-centric, event-driven, adaptive, working in volatile environments etc.;

Seventh dimension (user type): public administration staff, city citizens and humanbased organizations, machines-incl. vehicles, robots, and drones-, as well as connected cities;

Eighth dimension (interconnectivity type): wired vs. wireless vs. mobile vs. iBeacons vs. machine-to-machine vs. IoT, subsystem to subsystem, system to system, system of one city to system of another city, city to city-i.e. smart complete networking;

Ninth dimension (systemic characteristics): performance-incl. responsiveness, efficiency, and effectiveness-, quality-incl. reliability-, multifunctionality, interoperability-incl. interconnectivity-, complexity, modularity, scalability, adaptability-incl. configurability and non-obsolescence-, autonomy, resilience, transparency, data openness, coparticipation-incl. crowdsourcing-, security and privacy exposure, degree of automationincl. AI-, predictability etc.

By acknowledging all these dimensions, it is hard to propose a visionary model for assuring the best and shortest implementation path for transforming cities into smarter cities. The identification of a set of models to handle typical city situations could be the subject of another research, i.e. to indicate based on experience, experiments, and computed scores which are the best technological solutions, project setups, and roadmaps to develop the cities in an efficient and effective manner. Thereby, the components of the nine dimensions could be quantified in various axes-e.g. competence to implement/manage/maintain, budget to implement, time to implement, possibilities of integration to other standard systems and then assembled to create the models. The associated results of this research could also be used, besides the cities themselves, by the international/national standardization bodies to decide upon the priorities to define the most critical standards needed by the world, because if we consider the important topic of smart city applications, the review of international standards clearly shows that the needed elements, integration, and processes are only partially crystalized and framed at present.

Given this complexity and the fact that the overall status-quo-e.g. culture, legislation, resources, context is very different from one city of the world to another, it is hard to provide a straightforward and generally valid response for the three questions from above (Q1, Q2, Q3). All outlined aspects make the idea that each smart city must tailor and speed up their smart transformation by themselves plausible, by following for compatibility and intra-/interconnectivity reasons, as much as possible, the related international standards and by letting the door open for future upgrades, recalibrations, reconfigurations, adaptations, integration of new systems, and smart collaboration with other cities.

A synthetized response for Q1 is that the minimal smart components for a rapid transformation and optimum functional level should be found by a thorough deep collaborative analysis for each city conducted by multidisciplinary experts. With respect to Q2, it is clear that each existing international/national standard brings a series of benefits, but there could be inherent disadvantages for adopting fixed solutions, because some could not fit to the local 
conditions or could induce bigger specific risks-e.g. cyber-threats, therefore, this subject must be also treated competently, similarly to Q1 case. Finally, to answer Q3, it is certain that cities cannot wait too much, so as not to confront themselves with economic, social, territorial, political, or environmental bottlenecks, jams, disasters or even points of no return, conclusively, they must address and start implementing Q1 and Q2 as soon as possible.

As for the community's position regarding smart city solutions, it is clear that it depends to a high extent on the local status-quo, e.g. the overall positive survey results mentioned in the previous chapter may be correlated with the fact that Timis is one of the most advanced counties in Romania, according to all official KPI. The situation can be though improved anytime and anywhere by informing, training, and involving the residents in the actual steps of their conceptualization, implementation, and operation.

A way to handle this complexity of factors is to own or hire a research-developmentinnovation center-RDI center, for developing and deploying the necessary specific smart solutions. Moreover, in terms of joining additional forces, hiring businesses and other stakeholders in various forms of collaboration such as public-private partnerships is essential (Osborne Clarke, 2015, Emerson et al., 2012). Private companies can faster stimulate and guide the transformation of cities into smarter cities, according to their own economic interests, which must be in a win-win relation with the interests of the local community.

All in all, the envisioned solutions should consider all fore-mentioned aspects and the human-machine dependability balance, so that people and societies develop harmoniously, healthily, and without risks.

\section{Limitations and Suggestions for Future Research}

Each of the four results subchapters of this paper indicates existing gaps and further research actions which are implied or proposed by the authors.

\section{Conclusions}

Smart systems to be deployed in the cities of twenty-first century are very complex, therefore, they must be characterized by inspired visions, competent strategies, high competencies, and wise developments. Smart city systems should be protective and resilient, and, furthermore, they should be designed in such a way that they learn and adapt their behavior to current realities and necessities.

Some smart city international standards at different abstraction levels are under development or already exist, but unfortunately certain important aspects are not covered at present. More questions rise, with respect to what set of minimal smart components should be installed in the cities?, how much standardized the solutions should be?, and how much time cities should wait for their full standardization and technological development?, given the high pressure which is manifesting in multiple spheres of day by day life.

A good way to overcome some of the actual issues, take the upcoming opportunities, learn, and evolve faster, is to interconnect the cities themselves by smart common platforms. However, this solution implies that their internal systems are compatible, therefore, developed with this purpose in mind from the beginning.

Nine major dimensions of smart city systems identified by this paper make the selection of development choices very difficult for governors, and, if one adds the complexity of local status-quos, as well as the necessity of obtaining local community's and direct stakeholders' acceptance, the challenges are then acknowledged at their full amplitude.

Therefore, the collaboration with RDI centers and private partners seems to be a necessity, as solutions must fit to all beforementioned factors, which are ever changing in the globalizing world. As such, all requirements of 
smart city systems must be very well identified, designed, implemented, deployed, maintained, adapted, and improved permanently.

All in all, by comprehending all aspects mentioned before, a macrosystemic top-down approach for developing smart cities, whilst considering the local specificities, would clearly bring benefits to those cities on the long run.

\section{Acknowledgements}

This research did not receive any support from third parties.

\section{References}

1. Abbas, N., Zhang, Y., Taherkordi, A. and Skeie, T. (2017) 'Mobile edge computing: A survey,' IEEE Internet of Things Journal, 5 (1), 450-465.

2. Adio-Moses, D. A. and Asaolu, O. S. (2016), 'Artificial intelligence for sustainable development of intelligent buildings' Proceedings of the CIDB Postgraduate Conference, 2-4 February 2016, Cape Town, South Africa: University of Cape Town.

3. AFNOR. (2015). Smart and Sustainable Cities and Communities Coordination Group - Final report. Available: http://www.etsi.org/images/files/SSCCCG Final Reportrecommendations Jan 2015.pdf.

4. Agarwal, P. K., Gurjar, J., Agarwal, A. K. and Birla, R. (2015) 'Application of artificial intelligence for development of intelligent transport system in smart cities,' International Journal of Transportation Engineering and Traffic System, 1 (1), 20-30.

5. Ahuja, A. (2016) Integration of Nature and Technology for Smart Cities, Springer, Cham, Switzerland.

6. Alexander, J. C., Thompson, K. and Edles, L. D. (2016) Contemporary Introduction to Sociology: Culture and Society in Transition, Taylor \& Francis, USA.
7. Angelidou, M., Angelidou, M., Psaltoglou, A., Psaltoglou, A., Komninos, N., Komninos, N., Kakderi, C., Kakderi, C., Tsarchopoulos, P. and Tsarchopoulos, P. (2017) 'Enhancing sustainable urban development through smart city applications,' Journal of Science and Technology Policy Management.

8. Apolinarski, W., Iqbal, U. and Parreira, J. X. 'The GAMBAS middleware and SDK for smart city applications' Proceedings of the International Conference on Pervasive Computing and Communications (PerCom) ISBN: 1479927368, 24-28 March 2014, Budapest, Hungary: IEEE, 117-122.

9. Arafah, Y. and Winarso, $\mathrm{H}$. 'Redefining smart city concept with resilience approach' Proceedings of the International Conference of Planning in the Era of Uncertainty (ICPEU), ISBN: 1755-1315, 6-7 March 2017, Malang, Indonesia: IOP Publishing.

10. Arfat, Y., Aqib, M., Mehmood, R., Albeshri, A., Katib, I., Albogami, N. and Alzahrani, A. (2017) 'Enabling Smarter Societies through Mobile Big Data Fogs and Clouds,' Procedia Computer Science, 109, 1128-1133.

11. Arup \& Qualcomm. (2016). The Future of Connected Streets. Available: https://www.qualcomm.com/media/docume $\underline{\text { nts/files/the-future-of-connected-streets.pdf. }}$

12. Ballon, P., Glidden, J., Kranas, P., Menychtas, A., Ruston, S. and Graaf, S. v. d. 'Is there a Need for a Cloud Platform for European Smart Cities?' Proceedings of the eChallenges e-2011, 26-28 Octomber 2011, Florence, Italy: International Information Management Corporation, 1-7.

13. Bangui, H., Buhnova, B., Rakrak, S. and Raghay, S. (2017), 'Smart mobile technologies for the city of the future' Proceedings of the Smart City Symposium Prague (SCSP), ISBN: 1538638258, 25-26 May 2017, Prague, Czech Republic: IEEE, 1-6.

14. Barns, S. (2018) 'Smart cities and urban data platforms: Designing interfaces for 
smart governance,' City, Culture and Society, $12,5-12$.

15. Beurden, J. B.-v., Kallaos, J., Gindroz, B., Riegler, J., Noll, M., Costa, S. and Maio, R. (2017). Smart city guidance package for integrated planning and management. Available: $\quad \underline{\text { https://eu- }}$ smartcities.eu/sites/default/files/201709/SCGP\%20Intermediate\%20version \%20Ju $\underline{\text { ne} \% 202017 . p d f}$.

16. Bhatt, V., Brutti, A., Burns, M. and Frascella, A. (2017), 'An approach to provide shared architectural principles for interoperable smart cities' Proceedings of the International Conference on Computational Science and Its Applications (ICCSA), ISBN: 978-3-319-62398-6 (online), 978-3-31962397-9 (print), 3-6 July 2017, Trieste, Italy: Springer, 415-426.

17. Bibri, S. E. and Krogstie, J. (2017) 'Smart sustainable cities of the future: An extensive interdisciplinary literature review,' Sustainable Cities and Society, 31, 183-212.

18. Bolívar, M. P. R. and Meijer, A. J. (2016) 'Smart Governance: Using a Literature Review and Empirical Analysis to Build a Research Model,' Social Science Computer Review, 34 (6), 673-692.

19. Casini, M. (2016) Smart Buildings: Advanced Materials and Nanotechnology to Improve Energy-Efficiency and Environmental Performance, Woodhead Publishing.

20. Cicirelli, F., Guerrieri, A., Spezzano, G. and Vinci, A. (2017) 'An edge-based platform for dynamic Smart City applications,' Future Generation Computer Systems, 76, 106-118.

21. Connolly, D., Lund, H. and Mathiesen, B. (2016) 'Smart Energy Europe: The technical and economic impact of one potential $100 \%$ renewable energy scenario for the European Union,' Renewable and Sustainable Energy Reviews, 60, 1634-1653.

22. Di Martino, B., Li, K.-C., Yang, L. T. and Esposito, A. (eds.) (2017), Internet of Everything: Algorithms, Methodologies,
Technologies and Perspectives, Springer, Singapore.

23. Dijk, A. v. and Teuben, H. (2015). Smart Cities: How rapid advances in technology are reshaping our economy and society. Version 1.0. Available: https://www2.deloitte.com/content/dam/De loitte/tr/Documents/public-sector/deloittenl-ps-smart-cities-report.pdf.

24. Emerson, K., Nabatchi, T. and Balogh, S. (2012) 'An Integrative Framework for Collaborative Governance,' Journal of Public Administration Research and Theory, 22 (1), 129.

25. European Telecommunications Standards Institute. (2015). ETSI TR 103290 Machine-to-Machine communications (M2M); Impact of Smart City Activity on IoT Environment. TR 103 290. Available: http://www.etsi.org/deliver/etsi_tr/103200 $103299 / 103290 / 01.01 .0160 / \operatorname{tr} 103290 v 01$ 0101p.pdf.

26. Fernández-Güell, J.-M., Collado-Lara, M., Guzmán-Araña, S. and Fernández-Añez, V. (2016) 'Incorporating a systemic and foresight approach into smart city initiatives: the case of Spanish cities,' Journal of Urban Technology, 23 (3), 43-67.

27. Ferraz, F. S., Ferraz, C. A. G. and Gomes, A. 'Smart Cities Security Issues: An Impeding Identity Crisis' Proceedings of the International Conference on Software Engineering Advances (ICSEA), 21-25 August 2016, Rome, Italy: IARIA, 302-310.

28. Ferrer, J. N., Costa, S., Chira, C., Deambrogio, E., Horatz, M., Lindholm, P., Nielsen, D., Pasic, E. and Bhana, R. (2013a). Smart Cities Stakeholder Platform: Using EU funding mechanism for Smart Cities. Available:

http://www.tecniberia.es/documentos/Guid eline Using EU fundings mechanism for sma rt cities.pdf.

29. Ferrer, J. N., Olivero, S., K. MedarovaBergstorm and Rizos, V. (2013b). Smart Cities Stakeholder Platform: Financing models for smart cities. 2.0. Available: 
http://www.uraia.org/documents/109/2013 - Smart Cities Platform European Union Financing Models-ENG.pdf.

30. Garcia Lopez, P., Montresor, A., Epema, D., Datta, A., Higashino, T., Iamnitchi, A., Barcellos, M., Felber, P. and Riviere, E. (2015) 'Edge-centric computing: Vision and challenges,' ACM SIGCOMM Computer Communication Review, 45 (5), 37-42.

31. Goldsmith, S. and Crawford, S. (2014) The Responsive City: Engaging Communities Through Data-Smart Governance, JosseyBass, San Francisco, California.

32. Gubbi, J., Buyya, R., Marusic, S. and Palaniswami, M. (2013) 'Internet of Things (IoT): A vision, architectural elements, and future directions,' Future generation computer systems, 29 (7), 1645-1660.

33. Habibzadeh, M., Xiong, W., Zheleva, M., Stern, E., Nussbaum, B. and Soyata, T. (2017), 'Smart City Sensing and Communication Sub-Infrastructure' Proceedings of the International Midwest Symposium on Circuits and Systems (MWSCAS), 06-09 August 2017, Boston, Massachusetts, 1159-1162.

34. Hancke, G. P. and Hancke Jr, G. P. (2012) 'The role of advanced sensing in smart cities,' Sensors, 13 (1), 393-425.

35. Hashem, I. A. T., Chang, V., Anuar, N. B., Adewole, K., Yaqoob, I., Gani, A., Ahmed, E. and Chiroma, H. (2016) 'The role of big data in smart city,' International Journal of Information Management, 36 (5), 748-758.

36. Helfert, M., Krempels, K.-H., Klein, C., Donnellan, B. and Gusikhin, O. (eds.) (2016), Smart Cities, Green Technologies, and Intelligent Transport Systems: 4th International Conference, SMARTGREENS 2015, and 1st International Conference VEHITS 2015, Lisbon, Portugal, May 20-22, 2015, Revised Selected Papers, Springer.

37. Hessel, V. (2014). Smart Cities: A Siemens perspective. Available: http://www.clines- project.eu/gestor/recursos/uploads/archivos /Siemens VolkerHessel CLINES 13Jun14.pdf.

38. Huawei. (2017). Harnessing the Power of Connectivity: Mapping your transformation into a digital economy with GCI 2017. Available: http://www.huawei.com/minisite/gci/files/g ci 2017 whitepaper en.pdf.

39. Hurwitz, J., Kaufman, M. and Bowles, A. (2015) Smarter Cities: Cognitive Computing in Government. Cognitive Computing and Big Data Analytics, Wiley, USA and Canada.

40. IEC. (2014). Orchestrating infrastructure for sustainable Smart Cities. IEC WP Smart Cities: 2014-11(en). Available: www.iec.ch/whitepaper/pdf/iecWPsmartcities-LR-en.pdf.

41. IEC. (2019a). About the IEC $>$ What we do $>$ Technology sectors $>$ Smart Cities. [Online]. IEC. [Retrieved January 07, 2019]. Available:

http://www.iec.ch/smartcities/introduction. htm.

42. IEC. (2019b). SyC Smart Cities Electrotechnical aspects of Smart Cities. [Online]. IEC. [Retrieved January 07, 2019]. Available:

http://www.iec.ch/dyn/www/f?p=103:186:9 101923858123::::FSP ORG ID:13073.

43. IEEE Staff (ed.) (2016), 2016 International Conference on Security of Smart Cities, Industrial Control System and Communications (SSIC), IEEE.

44. IEEE Standards Association. (2016). IEEE Standards Activities for Smart Cities. Available:

http://standards.ieee.org/develop/msp/sma rtcities.pdf.

45. IEEE Standards Association. (2017). IEEE Standards Help Enable Smart City Technologies for Humanity. 059-3-17. Available: https://beyondstandards.ieee.org/wpcontent/uploads/2017/04/IEEE Smart City. pdf. 
46. International Electrotechnical Commission. (2016). IoT 2020: Smart and secure IoT platform. IEC WP IoT Platform:2016-10(en). Available: http://www.iec.ch/whitepaper/pdf/iecWPloT2020-LR.pdf.

47. International Telecommunication Union. (2015). Cybersecurity, data protection and cyber resilience in smart sustainable cities. Available: https://www.itu.int/en/ITU-

$\mathrm{T} /$ focusgroups/ssc/Documents/website/web -fg-ssc-0090-r7-

technical report on ICT infrastructure for re silience security.doc.

48. ISO. (2015). ISO/IEC JTC 1: Smart Cities. Preliminary Report 2014. Available: https://www.iso.org/files/live/sites/isoorg/f iles/developing standards/docs/en/smart ci ties_report-jtc1.pdf.

49. ISO. (2019a). Standards catalogue: ISO/IEC JTC 1/SC 41 - Internet of Things and related technologies. [Online]. ISO. [Retrieved January 07, 2019]. Available: https://www.iso.org/committee/6483279/x /catalogue/p/1/u/1/w/0/d/0.

50. ISO. (2019b). Standards catalogue: ISO/TC 268 - Sustainable cities and communities. [Online]. ISO. [Retrieved January 07, 2019]. Available: https://www.iso.org/committee/656906/x/c atalogue/p/1/u/1/w/1/d/1.

51. ISO. (2019c). Standards catalogue: ISO/TC 268/SC 1 - Smart community infrastructures. [Online]. ISO. [Retrieved January 07, 2019]. Available: https://www.iso.org/committee/656967/x/c atalogue $/ \mathrm{p} / 1 / \mathrm{u} / 1 / \mathrm{w} / 0 / \mathrm{d} / 0$.

52. ISO. (2019d). Standards catalogue: ISO/TC 301 - Energy management and energy savings. [Online]. ISO. [Retrieved January 07, 2019]. Available: https://www.iso.org/committee/6077221/x $/$ catalogue $/ \mathrm{p} / 1 / \mathrm{u} / 1 / \mathrm{w} / 0 / \mathrm{d} / 0$.

53. ITU. (2015). Standardization roadmap for smart sustainable cities. Available: $\quad$ https://www.itu.int/en/ITU-
T/focusgroups/ssc/Documents/website/web -fg-ssc-0274-r4-

technical report standardization roadmap.do c.

54. ITU. (2019a). ITU-T Recommendations under Study Group 20 responsibility. [Online]. ITU-T. [Retrieved January 07, 2019]. Available: https://www.itu.int/ITU-

$\mathrm{T} /$ recommendations/index sg.aspx?sg=20.

55. ITU. (2019b). Technical papers and technical reports. [Online]. ITU-T. [Retrieved January 07, 2019]. Available: https://www.itu.int/pub/T-TUT.

56. Jokinen, J., Latvala, T. and Lastra, J. L. M. 'Integrating smart city services using Arrowhead framework' Proceedings of the Annual Conference of the IEEE Industrial Electronics Society (IECON), ISBN: 1509034749, 23-27 October 2016, Florence, Italy: IEEE, 5568-5573.

57. Joss, S., Cook, M. and Dayot, Y. (2017) 'Smart cities: Towards a new citizenship regime? A discourse analysis of the British Smart City Standard,' Journal of Urban Technology, 24 (4), 29-49.

58. Kar, A. K., Gupta, M. P., Ilavarasan, P. V. and Dwivedi, Y. K. (eds.) (2017), Advances in smart cities : smarter people, governance, and solutions, CRC Press, Boca Raton, Florida.

59. Khan, Z., Anjum, A., Soomro, K. and Tahir, M. A. (2015) 'Towards cloud based big data analytics for smart future cities,' Journal of Cloud Computing, 4 (1), 2.

60. Kumar, T. M. V. (ed.) (2016), Smart Economy in Smart Cities: International Collaborative Research: Ottawa, St.Louis, Stuttgart, Bologna, Cape Town, Nairobi, Dakar, Lagos, New Delhi, Varanasi, Vijayawada, Kozhikode, Hong Kong, Springer, Singapore.

61. Kumar, T. M. V. (ed.) (2017), EDemocracy for Smart Cities, Springer, Singapore. 
62. Lea, R. (2017). Smart Cities: An Overview of the Technology Trends Driving Smart Cities. Available: https://www.ieee.org/publications_standard s/publications/periodicals/ieee-smart-citiestrend-paper-2017.pdf.

63. Mao, Y., You, C., Zhang, J., Huang, K. and Letaief, K. B. (2017) 'A survey on mobile edge computing: The communication perspective,' IEEE Communications Surveys \& Tutorials, 19 (4), 2322-2358.

64. Marcos Paramio, T. (2017). Abril 2017 - Normas UNE publicadas y proyectos PNE en elaboración del CTN 178. Available: https://www.clubdeinnovacion.es/images/in formes/Normas $\% 20 \mathrm{y} \% 20$ proyectos $\% 20 \mathrm{CTN}$ \%20178\%20-\%20Abril\%202017.pdf.

65. Martínez, M. L., Palomo, Á., Gil, O. and Navío, J. (2016) Impact Analysis of Smart City Networks in Cities' Local Government: RECI's Case, Colegio Oficial de Ingenieros de Telecomunicación, Madrid, Spain.

66. Mathur, S. and Modani, U. S. (2016), 'Smart City-a gateway for artificial intelligence in India' Proceedings of the Students' Conference on Electrical, Electronics and Computer Science (SCEECS), ISBN: 1467379182, 5-6 March 2016, Bhopal, India: IEEE, 1-3.

67. Merlino, G., Bruneo, D., Distefano, S., Longo, F., Puliafito, A. and Al-Anbuky, A. (2015) 'A smart city lighting case study on an openstack-powered infrastructure,' Sensors, 15 (7), 16314-16335.

68. Mohamed, N., Al-Jaroodi, J., Jawhar, I., Lazarova-Molnar, S. and Mahmoud, S. (2017) 'SmartCityWare: A Service-Oriented Middleware for Cloud and Fog Enabled Smart City Services,' IEEE Access, 5, 17576-17588.

69. Mrówczyńska, M., Alsabry, A., Skiba, M. and Bazan-Krzywoszańska, A. (2014) 'Research on energy consumption in the city. A system of modelling potential for energy effectiveness using artificial intelligence,' International Journal of Engineering Science and Technology, 3 (5), 373-384.
70. Nguyen, M. T. and Boundy, E. (2017) Big Data and Smart (Equitable) Cities. In: Thakuriah, P., Tilahun, N. \& Zellner, M. (eds.), Seeing Cities Through Big Data: Research, Methods and Applications in Urban Informatics, Springer, Cham, Switzerland.

71. Nilsson, N. J. (2014) Principles of artificial intelligence, Morgan Kaufmann Publishers.

72. Odini, M.-P. (2016). Smart Cities. Smart Cars. Smart ...Living. Available: www.slideshare.net/mpodini/smart-citiessmart-cars-smart-living.

73. Orłowski, C., Ziółkowski, A., Orłowski, A., Kapłański, P., Sitek, T. and Pokrzywnicki, W. (2016) Implementation of business processes in smart cities technology. In: Nguyen N.T., R., K., Orlowsky, C. \& Ziolkowski, A. (eds.), Transactions on Computational Collective Intelligence $\mathrm{XXV}$, Springer, Berlin, Germany.

74. Osborne Clarke. (2015). Smart cities in Europe: Enabling innovation. 23478386. Available:

http://www.cleanenergypipeline.com/Resou rces/CE/ResearchReports/Smart $\% 20$ cities $\%$ 20in\%20Europe.pdf.

75. Palomo-Navarro, Á. and Navío-Marco, J. (2017) 'Smart city networks' governance: The Spanish smart city network case study,' Telecommunications Policy.

76. Pan, Y. (2016) 'Heading toward artificial intelligence 2.0,' Engineering, 2 (4), 409-413.

77. Pang, Z., Sun, L., Wang, Z., Tian, E. and Yang, S. (2016), 'A survey of cloudlet based mobile computing' Proceedings of the International Conference on Cloud Computing and Big Data (CCBD), ISBN: 1467383503, 0406 November 2015, Shanghai, China: IEEE, 268-275.

78. Papa, R. and Fistola, R. (eds.) (2016), Smart Energy in the Smart City: Urban Planning for a Sustainable Future, Springer. 
79. Pradeep Kumar, H. 2016. Multisensor-based Occupancy Monitoring for Energy Efficient Smart Buildings Based on Internet of Things. Master of Science, California State University.

80. Provan, K. G. and Kenis, P. (2008) 'Modes of network governance: Structure, management, and effectiveness,' Journal of public administration research and theory, 18 (2), 229-252.

81. Raaijen, T. and Daneva, M. 'Depicting the smarter cities of the future: A systematic literature review \& field study' Proceedings of the Smart City Symposium Prague (SCSP), ISBN: 1538638258, 25-26 May 2017, Prague, Czech Republic: IEEE, 1-10.

82. Ratti, C. and Claudel, M. (2016) The city of tomorrow: sensors, networks, hackers, and the future of urban life, Yale University Press, New Haven, London.

83. Sandip, R. and Debabrata, S. (2017) 'The Role of Cloud of Things in Smart Cities,' International Journal of Computer Science and Information Security, 14 (11).

84. Sethi, P. and Sarangi, S. R. (2017) 'Internet of Things: Architectures, Protocols, and Applications,' Journal of Electrical and Computer Engineering, 2017.

85. Shamsir, S., Mahbub, I., Islam, S. K. and Rahman, A. (2017), 'Applications of Sensing Technology for Smart Cities' Proceedings of the International Midwest Symposium on Circuits and Systems (MWSCAS), 06-09 August 2017, Boston, Massachusetts: IEEE.

86. Sigler, T. J. and Martinus, K. (2017) 'Extending beyond 'world cities' in World City Network (WCN) research: Urban positionality and economic linkages through the Australiabased corporate network,' Environment and Planning A: Economy and Space, 49 (12), 2916-2937.

87. Sinaeepourfard, A., Garcia, J., MasipBruin, X., Marín-Tordera, E., Cirera, J., Grau, G. and Casaus, F. (2016), 'Estimating Smart City sensors data generation' Proceedings of the
Mediterranean Ad Hoc Networking Workshop (Med-Hoc-Net), ISBN: 1509019839, 20-21 June 2016, Geltru, Spain: IEEE, 1-8.

88. Skouby, K. E. and Lynggaard, P. (2015), 'Smart home and smart city solutions enabled by 5G, IoT, AAI and CoT services' Proceedings of the International Conference on Contemporary Computing and Informatics (IC3I), ISBN: 1479966290, 27-29 November 2014, Mysore, India: IEEE, 874-878.

89. Smith, D. A. and Timberlake, M. (1995) 'Conceptualising and mapping the structure of the world system's city system,' Urban Studies, 32 (2), 287-302.

90. Sneps-Sneppe, M. and Namiot, D. (2016) 'On mobile cloud for smart city applications,' Computing Research Repository (preprint arXiv:1605.02886).

91. Soler, A. C. D. (2015) 'Going urban: marking cities, city networks and global commodity chains in the capitalist worldsystem,' International Journal of Political Science and Development, 3 (2), 108-118.

92. Song, H., Rawat, D. B., Jeschke, S. and Brecher, C. (eds.) (2017), Cyber-Physical Systems: Foundations, Principles and Applications, Academic Press, Switzerland.

93. Song, T., Kaleshi, D., Zhou, R., Boudeville, O., Ma, J.-X., Pelletier, A. and Haddadi, I. 'Performance evaluation of integrated smart energy solutions through large-scale simulations' Proceedings of the International Conference on Smart Grid Communications (SmartGridComm), ISBN: 1424496241, 17-20 Octomber 2011, Brussels, Belgium: IEEE, 37-42.

94. Strother, N. and Lawrence, M. (2016). IoT and the Future of Networked Energy: A Platform for Enhanced Energy Cloud Applications, Services, and Business Models. Available:

https://www.navigantresearch.com/research iot-and-the-future-of-networked-energy.

95. Taylor, E. and Rooney, T. (eds.) (2016), Surveillance Futures: Social and Ethical Implications of New Technologies for 
Children and Young People, Routledge, Abingdon (UK) and New York (USA).

96. Townsend, A. M. (2001) 'Network cities and the global structure of the Internet,' American Behavioral Scientist, 44 (10), 16971716.

97. Townsend, A. M. (2013) Smart cities : big data, civic hackers, and the quest for a new utopia, W.W. Norton \& Company, New York (USA) and London (UK).

98. Tranos, E. and Gertner, D. (2012) 'Smart networked cities?,' Innovation: The European Journal of Social Science Research, 25 (2), 175-190.

99. Trivedi, Y. (2016) 'Smart CitieS: StandardS Will enSure SuCCeSS,' IEEE Communications Magazine, 5.

100. Vasile, M. C. (2018) Smart Networking of Cities, a Must in the Global Paradigm, Int Business Information Management Assoc-Ibima, Norristown, Pennsylvania.

101. Vermesan, O. and Bacquet, J. (eds.) (2017), Cognitive Hyperconnected Digital Transformation: Internet of Things Intelligence Evolution, River Publishers, Aalborg, Denmark.

102. Vermesan, O. and Friess, P. (eds.) (2014), Internet of things-from research and innovation to market deployment, River Publishers, Aalborg, Denmark.

103. Vermesan, O. and Friess, P. (eds.) (2015), Building the hyperconnected society: IoT research and innovation value chains, ecosystems and markets, River Publishers, Aalborg, Denmark.

104. Vermesan, 0. and Friess, P. (eds.) (2016), Digitising the industry-internet of things connecting the physical, digital and virtual worlds, River Publishers, Gistrup, Denmark.

105. Vo, M.-T., Do, N.-H., Tran, V.-S., Ma, Q.K., Le, C.-T. and Mai, L. (2018), 'A multi-storey building actuator and sensor system using
6L0WPAN based Internet of Things: Practical design and implementation' Proceedings of the International Conference on Recent Advances in Signal Processing, Telecommunications \& Computing (SigTelCom), ISBN: 1538629763, 29-31 January 2018, Ho Chi Minh, Vietnam: IEEE, 176-181.

106. Walravens, N. and Ballon, P. (2013) 'Platform business models for smart cities: from control and value to governance and public value,' IEEE Communications Magazine 51 (6), 72-79.

107. Woods, E., Citron, R. and Strother, N. (2017). Leaderboard: Smart City Suppliers. Available:

https://www.navigantresearch.com/research Lnavigant-research-leaderboard-smart-citysuppliers.

108. Zhang, K. and Shen, X. (2015) Security and Privacy for Mobile Healthcare Networks, Springer, Cham, Switzerland. 